This is a post-peer-review, pre-copyedit version of a Journal article published in the Neural Computing and Applications

The final authenticated version is available online at:

https://link.springer.com/article/10.1007/s00521-019-04573-3 


\title{
Integrated computational intelligent paradigm for nonlinear electric circuit models using neural networks, genetic algorithms and sequential quadratic programming
}

\author{
Ammara Mehmood ${ }^{1}$, Aniela Zameer ${ }^{2}$, Sai Ho Ling, \#, Ata Ur Rehman ${ }^{4}$, a \\ Muhammad Asif Zahoor Raja, b
${ }^{1}$ Department of Electrical Engineering, Pakistan Institute of Engineering and Applied Sciences, Nilore, Islamabad, Pakistan
Email: ms_mehmud16@pieas.edu.pk
${ }^{2}$ Department of Computer and Information Sciences, Pakistan Institute of Engineering and Applied Sciences, Nilore, Islamabad, Paksitan \\ Emails: aneelaz@pieas.edu.pk, \\ ${ }^{3}$ School of Biomedical Engineering, Centre for Health Technologies, Department of Engineering and IT, University \\ of Technology, Sydney, NSW, Australia \\ Steve.Ling@uts.edu.au \\ ${ }^{\#}$ Corresponding author \\ ${ }^{4}$ Department of Electrical and Computer Engineering, COMSATS University Islamabad, Attock Campus, Attock, \\ Pakistan \\ aEmail: Muhammad.asif@ciit-attock.edu.pk \\ bEmail: dr.ataurrehman@ciit-attock.edu.pk
}

\begin{abstract}
In this paper, a novel application of biologically inspired computing paradigm is presented for solving initial value problem (IVP) of electric circuits based on nonlinear RL model by exploiting the competency of accurate modeling with feed forward artificial neural network (FF-ANN), global search efficacy of Genetic algorithms (GA), and rapid local search with sequential quadratic programming (SQP). The fitness function for IVP of associated nonlinear RL circuit is developed by exploiting the approximation theory in mean squared error sense using an approximate FF-ANN model. Training of the networks is conducted by integrated computational heuristic based on GA aided with SQP, i.e., GA-SQP. The designed methodology is evaluated to variants of nonlinear RL systems based on both $\mathrm{AC}$ and $\mathrm{DC}$ excitations for number of scenarios with different voltages, resistances and inductance parameters. The comparative studies of the proposed results with Adams numerical solutions in terms of various performance measures verify the accuracy of the scheme. Results of statistics based on MonteCarlo simulations validate the accuracy, convergence, stability and robustness of the designed scheme for solving problem in nonlinear circuit theory.
\end{abstract}

Keywords: Artificial neural networks; Nonlinear systems; Nonlinear electric circuits; Genetic algorithms; Sequential quadratic programming. 


\section{Nomenclature}

$\begin{array}{ll}\text { AE } & \text { Absolute Error } \\ \text { ANS } & \text { Adam numerical solver } \\ \text { DC/AC } & \text { Direct Current/ Alternating Current } \\ \text { EGNSE } & \text { error function of NSE } \\ \text { EVAF } & \text { error function of VAF } \\ \text { FF-ANN } & \text { forward artificial neural network } \\ \text { GA } & \text { Genetic algorithms } \\ \text { GMAE } & \text { Global MAE } \\ \text { GNSE } & \text { Global NSE } \\ \text { GVAF } & \text { Global VAF } \\ \text { IVP } & \text { initial value problem } \\ \text { MAE } & \text { Mean Absolute Error } \\ \text { MIN } & \text { minimum } \\ \text { NNDEM } & \text { neural network based differential equations models } \\ \text { NSE } & \text { Nash Sutcliffe efficiency } \\ \text { ODE } & \text { Ordinary Differential equations } \\ \text { RL } & \text { Resistor inductor } \\ \text { SQP } & \text { sequential quadratic programming } \\ \text { SS } & \text { stochastic solvers } \\ \text { STD } & \text { standard deviation } \\ \text { VAF } & \text { Variance account for } \\ i & \text { current } \\ \Psi & \text { flux-linkage of the inductor } \\ \alpha & \text { a constant } \\ \delta & \text { Unknown vector } \\ \boldsymbol{w} & \text { Unknown vector } \\ \boldsymbol{\beta} & \text { Unknown vector } \\ \varepsilon & \text { objective function } \\ & \end{array}$

\section{Introduction}

The universal function approximation strength of artificial neural networks (ANNs) has been utilized immensely by the researchers in diverse domain of engineering and technology [1-5]. For example, estimation of STATCOM voltages and reactive powers [6], optimization of heat conduction model of human head [7], optimization of an irreversible thermal engine [8], estimation of underwater inherent optical characteristics [9], prediction of attendance demand in games [10], nonlinear system based on elliptic partial differential equations [11] and optimization of credit classification analysis problems [12]. Recently, the use of stochastic solvers (SS) for effective solution of nonlinear systems based on differential equation has been reported broadly [13-16]. Few potential applications of SS for solution of differential equations include nonlinear optics studies [17], applications of random matrix theory [18], nonlinear stiff oscillatory systems based on Van der Pol oscillator [19], fuzzy nonlinear systems [20], magnetohydrodynamic problems [21], inverse kinematics problem [22], nonlinear Jeffery-Hamel 
flow model [23], parameter estimation [24], fuel ignition systems [25], fuzzy Fredholm-Volterra integrodifferential equations [26], nonlinear drainage problem based on Johnson-Segalman fluid [27], electrical conducting solids [28], nonlinear problems arising in nanotechnology [29], astrophysics [30], plasma physics [31], atomic physics [32], model of heartbeat dynamics [33], models of HIV infection of CD4+ T-cell model [34], fractional order systems [35], economic [36] and finance [37]. Additionally, analysis of nonlinear systems based on Thomas-Fermi [38], Lane-Emden [29], Emden-Fowler [40], Bratu [41], Troesch [42], Riccati [43], FlierlPetviashivili [44], Beglay-Torviq [45], Pantograph [46], Van der Pol [47] and Painlevé type equations [48] are other illustrative application of stochastics solvers. The competency of these methodologies to nonlinear problem arising in circuit theory can play a fundamental role due to unavailability of exact solution and strong nonlinearity in the governing mathematical models. Aim of the present study is to explore and exploit the field of an intelligent computing to design an accurate, reliable and robust stochastic solver to study the dynamics of Resistance-Inductance (RL) circuit with nonlinear inductance parameter.

In this study, integrated biologically inspired computational technique is designed for finding solution of nonlinear problems arising in electronic circuits using feed forward ANNs optimized initially with Genetic Algorithms (GAs) and refined by Sequential Quadratic Programming (SQP) technique. The basic schematic of RL circuits involving nonlinear inductor with DC/AC excitation is presented in Fig. 1 [49]

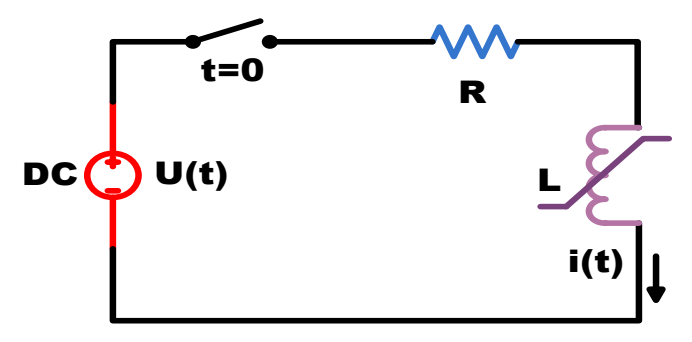

(a): Circuitry with DC excitation

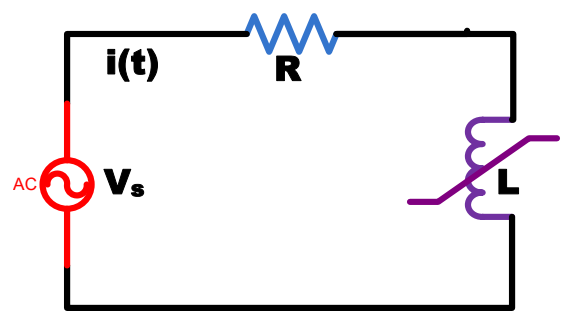

(b): Circuitry with AC excitation

Fig. 1: Schematic of RL circuits with nonlinear inductor

The mathematical formulation for the system model is described below:

$$
\psi^{\prime}+\alpha R \psi^{2}=U, \quad \psi(0)=0 .
$$

In RL circuits the branch relation of nonlinear inductor is written as:

$$
i(\psi)=\alpha \psi^{2},
$$

here $i$ is the current, $\Psi$ represent the flux-linkage of the inductor, and $\alpha$ stands for a constant. Now by applying Kirchhoff laws to RL circuit, one have

$$
\psi^{\prime}+R i(\psi)=u(t) .
$$

For DC excitation, i.e., $u(t)=U$, the equation (3) gives 


$$
\psi^{\prime}+\alpha R \psi^{2}=U, \quad \psi(0)=0
$$

By considering $\mathrm{AC}$ excitation, i.e., $u(t)=U_{m} \sin \omega t$

$$
\psi^{\prime}+\alpha R \psi^{2}=U_{m} \sin \omega t, \psi(0)=0
$$

The system represented in equations (4) and (5) has no exact solution, therefore comparative analysis of the proposed results has been executed with numerical experimentation results of Adams method. The salient features of the designed scheme include significant reduced effort is required to determine accurate results, easily extendable procedure to analogous system, simplicity of the concept, implementation ease, continuous solution within the input training span, less sensitivity to computational round off errors, and good alternative to solve stiff engineering problems which are remained challenging to traditional schemes.

The remaining paper is presented as, section 2 explains the proposed design methodology, section 3 describes the results of numerical experimentations and comparative study based on performance operators, while the paper is concluded in section 4.

\section{Designed Methodology}

The desired methodology comprises of two steps: the first part based on neural networks based differential equation modelling for the nonlinear system and then the merit function is constructed by approximation of an error function in the mean square sense, in the second phase, learning procedure is provided along with the definitions of performance indices for the evaluation of the results. Graphical illustration of the design scheme is shown in Fig. 2.

\subsection{Neural Network based Differential Equations Models}

The solution $\psi(t)$, its first $\psi^{\prime}$ and $n^{t h}$ order derivative $\psi^{(n)}$ in the form of neural network based differential equations models (NNDEM) for $n^{\text {th }}$ order ODE are approximated by the through continuous mapping in neural network methodology as:

$$
\left(\hat{\psi}, \hat{\psi}^{\prime}, \ldots, \hat{\psi}^{(n)}\right)=\left(\sum_{i=1}^{k} \delta_{i} f\left(w_{i} t+\beta_{i}\right), \sum_{i=1}^{k} \delta_{i} f^{\prime}\left(w_{i} t+\beta_{i}\right), \ldots, \sum_{i=1}^{k} \delta_{i} f^{(n)}\left(w_{i} t+\beta_{i}\right)\right)
$$

where $\delta, \boldsymbol{w}$ and $\boldsymbol{\beta}$ are the unknown vectors of $\boldsymbol{W}$ as:

$$
\boldsymbol{W}=[\boldsymbol{\delta}, \boldsymbol{w}, \boldsymbol{\beta}]=\left[\delta_{1}, \delta_{2}, \ldots, \delta_{m}, w_{1}, w_{2}, \ldots, w_{m}, \beta_{1}, \beta_{2}, \ldots, \beta_{m}\right]
$$

In NNDEM given in (6) generally developed using log-sigmoid, $f(z)=1 /\left(1+e^{-z}\right)$ as an activation function and its derivatives, therefore, the updated NNDEMs for the nonlinear RL circuits solutions is as follows: 


$$
\left(\hat{\psi}, \hat{\psi}^{\prime}\right)=\left(\sum_{i=1}^{k} \delta_{i} w_{i}\left(\frac{1}{1+e^{-\left(w_{i} t+\beta_{i}\right)}}\right), \sum_{i=1}^{k} \delta_{i} w_{i}\left(\frac{e^{-\left(w_{i} t+\beta_{i}\right)}}{\left(1+e^{-\left(w_{i} t+\beta_{i}\right)}\right)^{2}}\right)\right),
$$

The generic architecture of the NNDEMs for nonlinear RL circuit for DC and AC excitation can be formulated using set of equations (8).
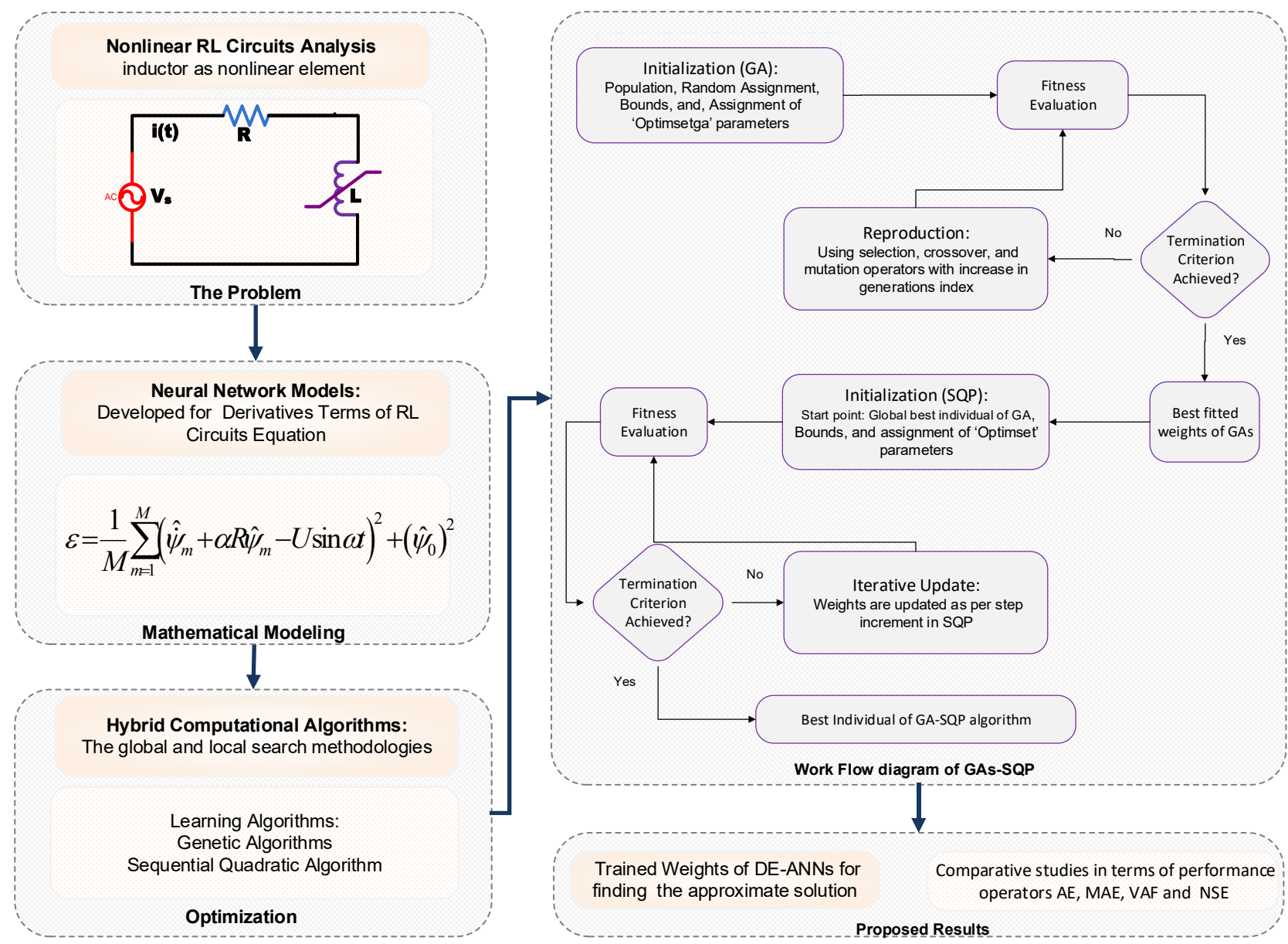

Fig 2. Generic workflow of hybrid computational method based on NNDEM optimized with GA and SQP

\subsection{Objective Functions}

The objective function $\varepsilon$ is the sum of the mean squared errors for training the optimized parameters for NNDEMs as:

$$
\varepsilon=\varepsilon_{1}+\varepsilon_{2}
$$

where $\varepsilon_{1}$ is an error function for DC excitation, while $\varepsilon_{2}$ is the error function formulated by considering the initial conditions. The elaborative form of equation (9) for RL based circuit models is given as: 


$$
\varepsilon=\frac{1}{M} \sum_{m=1}^{M}\left(\hat{\psi}_{m}^{\prime}+\alpha R \hat{\psi}_{m}-U\right)^{2}+\left(\hat{\psi}_{0}\right)^{2},
$$

while for AC excitation

$$
\varepsilon=\frac{1}{M} \sum_{m=1}^{M}\left(\hat{\psi}_{m}^{\prime}+\alpha R \hat{\psi}_{m}-U \sin \omega t_{m}\right)^{2}+\left(\hat{\psi}_{0}\right)^{2}
$$

for $\hat{\psi}_{m}=\hat{\psi}\left(t_{m}\right), t_{m}=m h, M=1 / h$,

Arbitrary unknown weights of NNDEM for which the value of error function $\varepsilon$ approaches ' 0 ' in case $\varepsilon$ approaches ' 0 ' for both DC and AC excitations and subsequently, the proposed approximate solution $\hat{\psi}(t)$ of the nonlinear circuit system is converges to exact solution $\psi(t)$. The standard form of DENNM based architecture for DC and AC excitations are shown in Figs. 3(a) and 3(b), respectively, while mathematically presented in respective equations (10) and (11). The DENNM are formulated based on single input layer, signal hidden layer with log-sigmoid, $f(z)=1 /\left(1+e^{-z}\right)$ as an activation function and signal output layer. Each operation for DENNM as presented in Figs. 3(a) and 3(b) are presented with different colours for better understanding.

\subsection{Learning of Weights for NNDEMs}

Learning methodology based on Genetic Algorithms (GAs) and Sequential Quadratic Programming (SQP) scheme is employed to minimize the objective function (9) by training of unknown parameters $\boldsymbol{W}$ of NNDEMs.

Global search competency of GAs is well-established in the class of initial population based optimization solvers of evolutionary computing domains. Mathematical foundations of GAs are built by inspiration of genetic mechanism and its the first application introduced by Holland in early 70's of nineteenth century [50]. Genetic algorithms operate on a randomly initialized population to produce improved approximations. Individuals are selected at each generation, from a pool of new population based on fitness level. At next step variation operators are applied. The algorithm is expertly used for convex/non-convex and constrained/unconstrained optimization problems having application in engineering and technology [51-54]. Few recently reported significance application includes are joint angle-amplitude estimation in direction of arrival of the plane waves [55], effective solution of traveling salesman problem [56], layer thickness optimization in multilayer piezoelectric transducer based systems [57], prediction of Thai stock price index trend [58] and nonlinear fuzzy systems [59] etc.

Hybridization with efficient local search methodologies is normally incorporated with GAs for speedy optimization of problem specific parameters. The effective constrained optimization solvers based on SQP algorithm is utilized for rapid local convergence of the variables. The necessary terms, theory, underlying concept, mathematical background, significance, implementation and applications of SQP, see [60-63]. Consequently, hybrid computing framework based on GAs and SQP is developed to get the optimization parameter of NNDEMs to solve nonlinear RL circuits with both AC/DC excitations. 


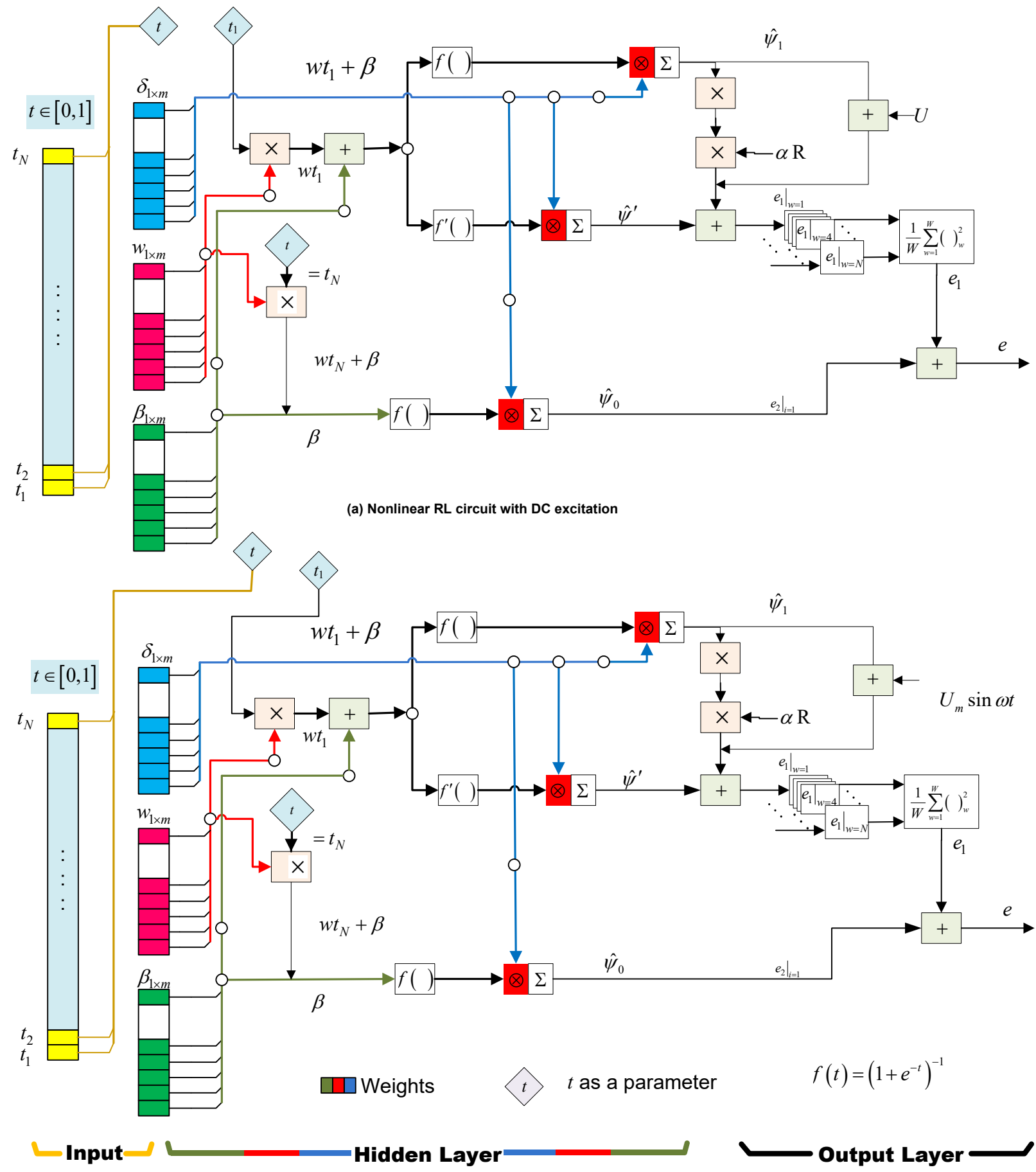

(b) Nonlinear RL circuit with AC excitation

Fig. 3 NNDEM design for nonlinear RL circuit for both DC and AC excitations.

The procedural steps of the GA-SQP algorithm is presented graphically in Fig 2, while elaborative explanation of GA in workflow diagram is represented in Fig. 4. In the Fig. 4, detailed description/operation of reproduction operators of GAs, i.e., selection, crossover and mutation, with graphical illustrations is provided. Additionally, the parameter settings incorporated for GA and SQP algorithm is provided in Table 1. Furthermore, the pseudocode of 
the proposed methodology GA-SQP to optimized the weights of NNDEM is given in Algorithm 1 for the ease in reproduction of the results. The performance of the algorithm is dependent of these settings, a slight change in these parameters may results in premature convergence. Therefore, a lot of care, experience and experimentation is required for selecting optimal parameters of meta-heuristics GA-SQP.

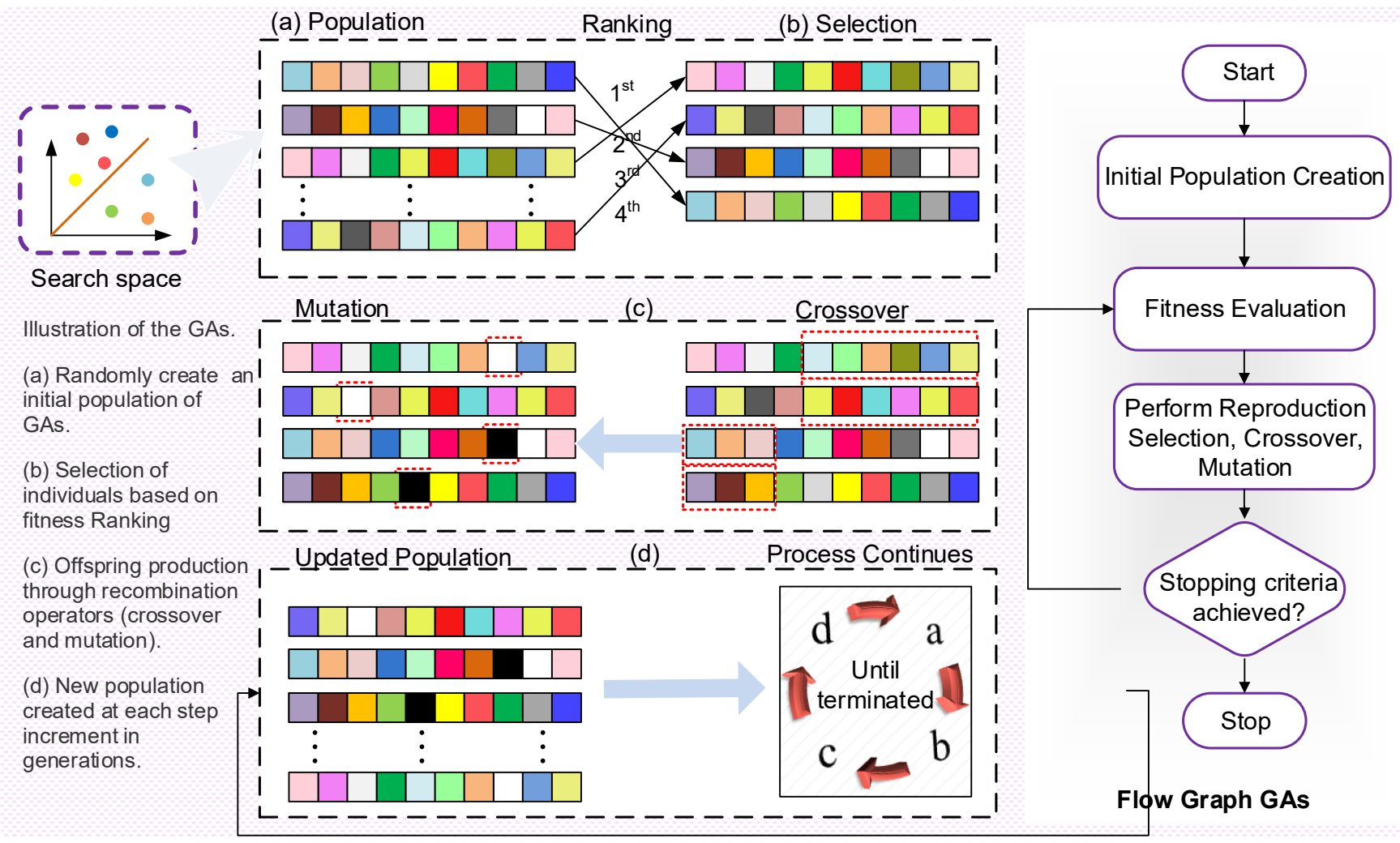

Fig. 4: Schematic workflow of Genetic Algorithms

Algorithm 1: Pseudocode of GA-SQP for solving circuit of nonlinear RL circuit model

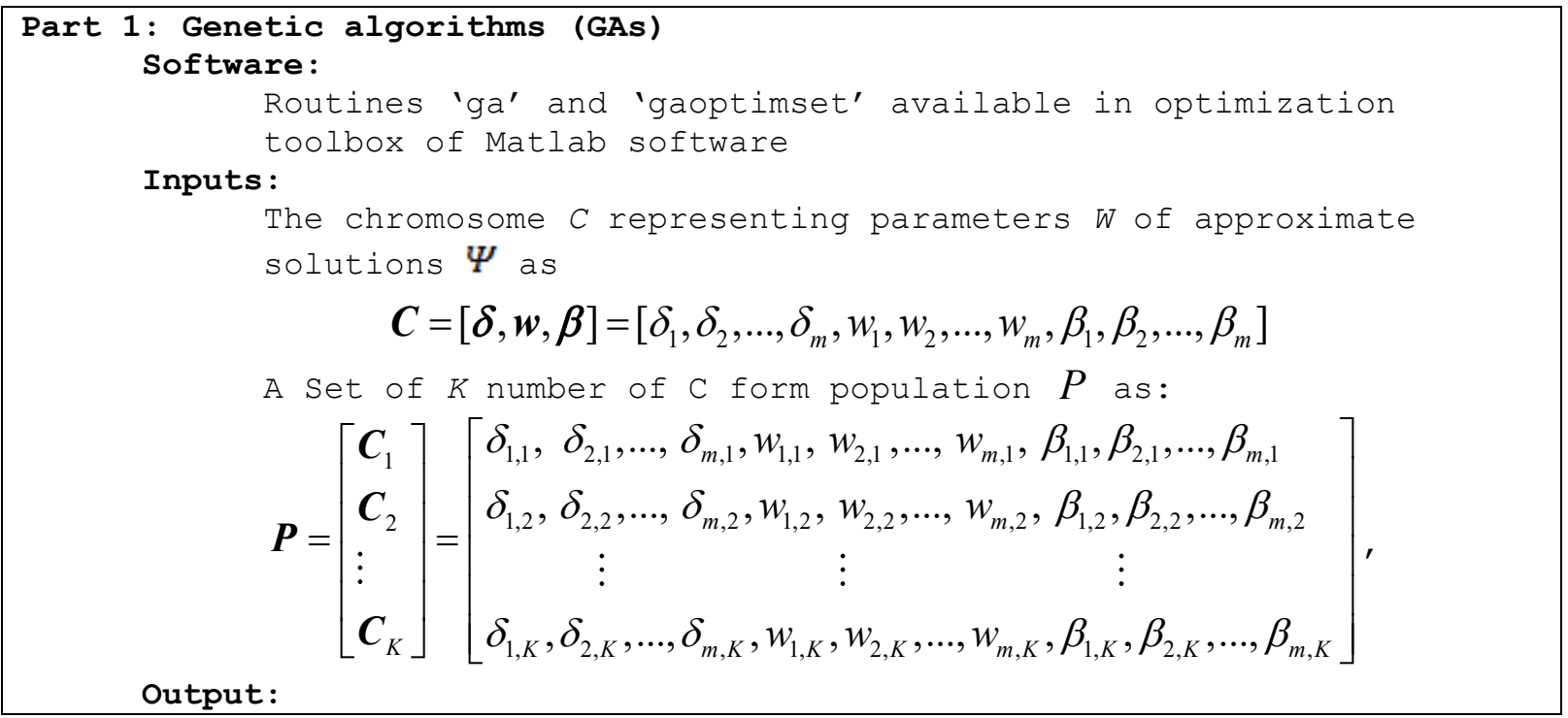




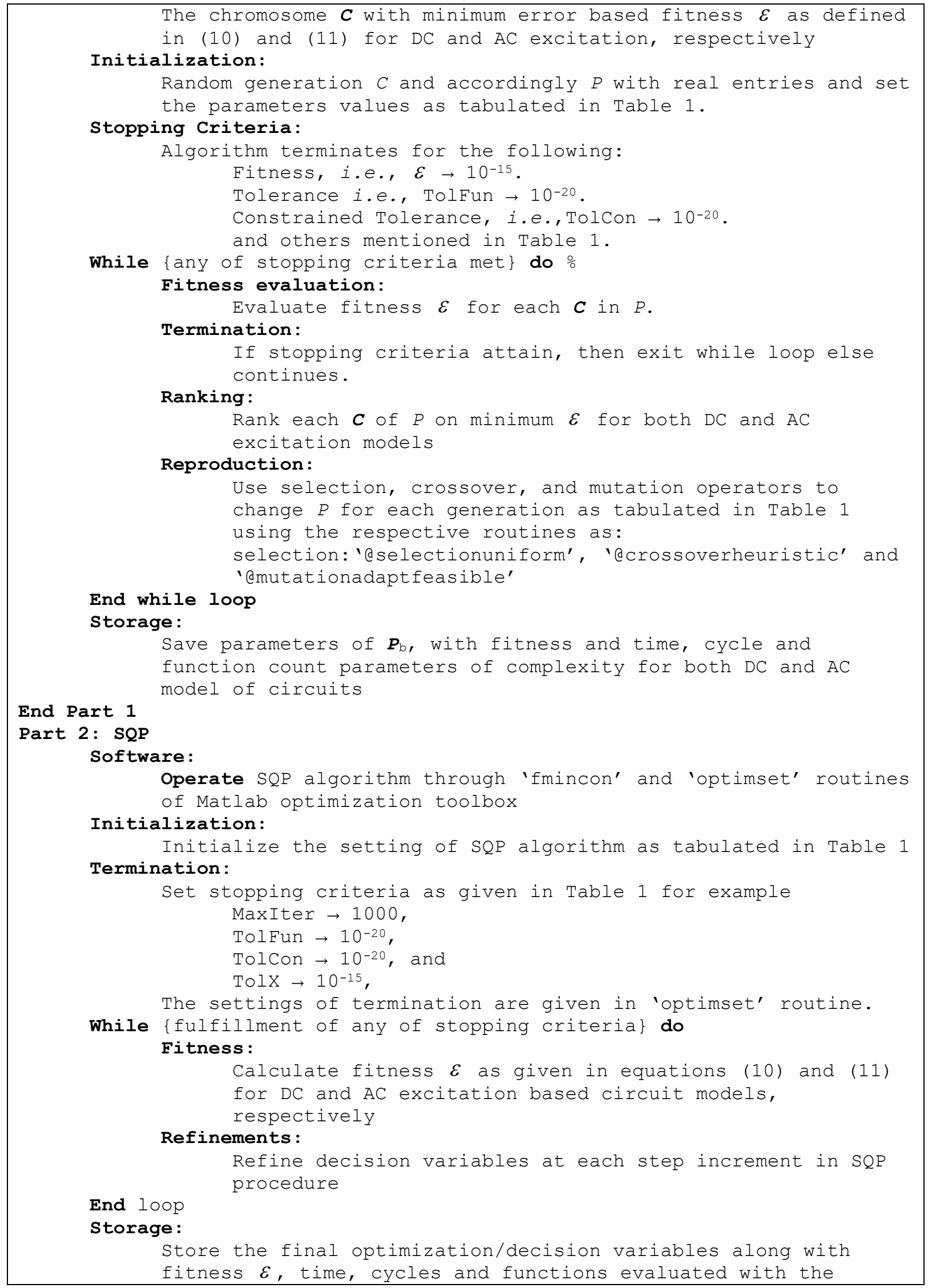




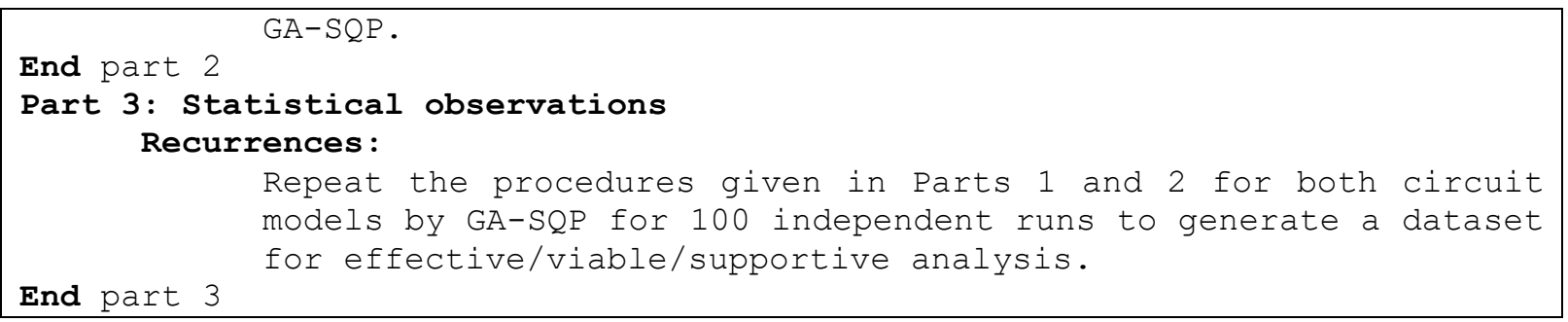

Table 1: Parameter Settings of GA and SQP methods

\begin{tabular}{l|lllll}
\hline Method & \multicolumn{1}{|c}{ Parameters } & \multicolumn{1}{c}{ Setting } & \multicolumn{1}{c}{ Parameters } & \multicolumn{1}{c}{ Setting } \\
\hline \hline GA & Population Creation & Constrained dependent & Population Size & 200 \\
& Scaling function & Rank & Variables & 30 \\
& Selection function & Stochastic Uniform & Generation & 400 \\
& Initial Population & {$[-1,1]$} & Function Tolerance & $10^{-15}$ \\
& Crossover function & Scattered & Stall Generation limit & 100 \\
& Mutation function & Adaptive feasible & Bounds (lower, upper) & $(-30,30)$ \\
& Elite count & 4 & Nonlinear Constraint Tolerance & $10^{-15}$ \\
SQP & Fitness Limit & $10^{-15}$ & Other & defaults \\
& Start Point & Best weights of GAs & Hessian & BFGS \\
& Derivative & Solver Approximate & Minimum Perturbation & $10-08$ \\
& Iterations & 1000 & X-Tolerance & $10-20$ \\
& Max. Function & 100000 & Nonlinear constraint Tolerance & $10-25$ \\
& evaluations & Function Tolerance & $10-25$ \\
& Bounds (lower, upper) & $(-30,30)$ & Other & Defaults \\
\hline \hline
\end{tabular}

\subsection{Performance Indices}

In this research study, performance of the designed scheme for solving nonlinear circuits models is examined by incorporating three different performance indices defined in terms of mean absolute error (MAE), Nash Sutcliffe efficiency (NSE), and variance account for (VAF). The advantages of using these three metrics is provision of in-depth analysis of the accuracy, convergence and stability on different optimal values for the perfect modeling.

The MAE metric provides the mean level of the model precision based of calculation of error from reference results along with the absolute operators that does not show the deviation direction of the error. Mathematically MAE and Global MAE (GMAE) for solution of nonlinear circuit model (1) are written as:

$$
(M A E, G M A E)=\left(\left(\frac{1}{M} \sum_{m=1}^{M}\left|\psi_{m}-\hat{\psi}_{m}\right|\right),\left(\frac{1}{R} \sum_{r}^{R}\left(\frac{1}{M} \sum_{m=1}^{M}\left|\psi_{m}-\hat{\psi}_{m}\right|\right)_{r}\right)\right)
$$

Besides MAE, the NSE metric, introduced by Nash and Sutcliffe, in 1970 [64] is a normalized statistic that shows a comparison between the relative magnitude of the residual variance and the measured data variance. NSE values ranges $[-\infty, 1]$ to determines the model efficiency and its 
value should be closer to 1 for perfect model. Mathematical relation for NSE and global NSE GNSE are described as:

$$
(N S E, G N S E)=\left(\left(1-\frac{\sum_{i=1}^{n}\left(\psi_{i}-\hat{\psi}_{i}\right)^{2}}{\sum_{i=1}^{n}\left(\psi_{i}-\bar{\psi}_{i}\right)^{2}}\right),\left(\frac{1}{K} \sum_{k=1}^{K}\left(1-\frac{\sum_{m=1}^{M}\left(\psi_{m}-\hat{\psi}_{m}\right)^{2}}{\sum_{m=1}^{M}\left(\psi_{m}-\bar{\psi}_{m}\right)^{2}}\right)_{k}\right)\right)
$$

and its error function $\mathrm{E}_{\mathrm{NSE}}$ and $\mathrm{EG}_{\mathrm{NSE}}$ are as follows:

$$
\left(E_{N S E}, E G_{N S E}\right)=\left((1-N S E),\left(\frac{1}{K} \sum_{k=1}^{K}(1-N S E)_{k}\right)\right)
$$

The VAF based performance metric is also used to verify the correctness of the model through comparison of actual output from approximate one. If they differ than VAF is lower. Mathematical relations for VAF and GVAF are defined as:

$$
(V A F, G V A F)=\left(\left(1-\frac{\operatorname{var}(\psi-\hat{\psi})}{\operatorname{var}(\psi)}\right), \frac{1}{k} \sum_{k=1}^{K}\left(1-\frac{\operatorname{var}(\psi-\hat{\psi})}{\operatorname{var}(\psi)}\right)_{k}\right)
$$

The error function linked with VAF (EVAF) is defined bellow:

$$
\left(E_{V A F}, E G_{V A F}\right)=\left((100-V A F),\left(\frac{1}{k} \sum_{k=1}^{K}(100-V A F)_{k}\right)\right)
$$

Magnitudes of MAE, $\mathrm{E}_{\mathrm{VAF}}$, and $\mathrm{E}_{\mathrm{NSE}}$ for perfect model are generally zero.

\section{Numerical experimentation with discussion}

Results of detailed numerical experimentations are given here for the solution two nonlinear RL circuit problems based on DC (1) and AC (2) excitations.

\subsection{Problem 1: Nonlinear RL circuit with DC excitation}

The dynamics of the problem is analyzed number of cases for three scenarios by varying the resistance $R$, voltage $U$ and inductance $\alpha$ parameters.

Scenario 1: Nonlinear RL circuit problem by varying the resistance parameter: In this study, a nonlinear RL based circuit model having constant values for $U=250$ and $\alpha=0.5$ parameters by varying the $R$ magnitudes are considered for the three cases, i.e., $R=125,100$ and 155 .

Scenario 2: Nonlinear RL circuit problem by varying the voltage parameter: In this study, a nonlinear RL based circuit model having constant values for $R=125$ and $\alpha=0.5$ parameters by varying the $U$ magnitudes are considered for the four cases, i.e., $U=150,200,250$ and 300 . 
Scenario 3: Nonlinear RL circuit problem by varying the inductance parameters: In this study, a nonlinear RL based circuit model having constant value for $U=150$ and $R=125$ parameters by varying the $\alpha$ magnitudes are considered for the four cases, i.e., $\alpha=0.75,1.0,1.5$ and 2.0.

\subsection{Problem 2: Nonlinear RL circuit with AC excitation}

The dynamics of the problem is analyzed number of cases for four scenarios by varying the resistance $R$, voltage $U$, inductance $\alpha$, angular frequency $\omega$ parameters.

Scenario 1: Nonlinear $R L$ circuit problem by varying the voltage parameter: In this study, a nonlinear RL based circuit model having constant values for $R=125, \alpha=0.5$ and $\omega=0.9$ parameters by varying the $U$ magnitudes are considered for the three cases, i.e., $U=150,100$ and 50 .

Scenario 2: Nonlinear $R L$ circuit problem by varying the resistance parameter: In this study, a nonlinear RL based circuit model having constant values for $U=100, \alpha=0.5$ and $\omega=0.9$ parameters by varying the $R$ magnitudes are considered for the three cases, i.e., $R=125,75$ and 175.

Scenario 3: Nonlinear RL circuit problem by varying the inductance parameters: In this study, a nonlinear RL based circuit model having constant values for $U=150, R=125$ and $\omega=0.9$ parameters by varying the $\alpha$ magnitudes are considered for the three cases, i.e., $\alpha=0.5,1.0$ and 1.5.

Scenario 4: Nonlinear $R L$ circuit problem by varying angular frequency: In this study, a nonlinear RL based circuit model having constant $U=150, R=125$ and $\alpha=0.5$. parameters by varying the $\omega$ magnitudes are considered for the three cases, i.e., $\omega=0.3,0.6$ and 0.9 .

Proposed scheme is executed for finding the solution of nonlinear RL circuit problems 1 and 2 for each case i.e., c-1 to c-4 for cases 1 to 4 , of all scenarios, i.e., s-1 to s-3 for scenarios 1 to 4 , as described in the last section. The objective functions as given in equations (10-11) for $M=10$ inputs grid points for case 1 of these scenarios for problem 1 based on DC excitation are expressed as:

$$
\begin{aligned}
& \varepsilon_{s-1, c-1}=0.1\left(\sum_{m=1}^{10}\left(\hat{\psi}_{m}^{\prime}+62.5 \hat{\psi}_{m}-250\right)^{2}\right)+\left(\hat{\psi}_{0}\right)^{2}, \\
& \varepsilon_{s-2, c-1}=0.1\left(\sum_{m=1}^{10}\left(\hat{\psi}_{m}^{\prime}+62.5 \hat{\psi}_{m}-150\right)^{2}\right)+\left(\hat{\psi}_{0}\right)^{2}, \\
& \varepsilon_{s-3, c-1}=0.1\left(\sum_{m=1}^{10}\left(\hat{\psi}_{m}^{\prime}+93.75 \hat{\psi}_{m}-150\right)^{2}\right)+\left(\hat{\psi}_{0}\right)^{2},
\end{aligned}
$$

Similarly, the objective functions for case 1 of four scenarios of problem 2 based on AC excitation are expressed as:

$$
\varepsilon_{s-1, c-1}=0.1\left(\sum_{m=1}^{10}\left(\hat{\psi}_{m}^{\prime}+62.5 \hat{\psi}_{m}-150 \sin (0.9) t_{m}\right)^{2}\right)+\left(\hat{\psi}_{0}\right)^{2},
$$




$$
\begin{aligned}
& \varepsilon_{s-2, c-1}=0.1\left(\sum_{m=1}^{10}\left(\hat{\psi}_{m}^{\prime}+62.5 \hat{\psi}_{m}-100 \sin (0.9) t_{m}\right)\right)+\left(\hat{\psi}_{0}\right)^{2}, \\
& \varepsilon_{s-3, c-1}=0.1\left(\sum_{m=1}^{10}\left(\hat{\psi}_{m}^{\prime}+62.5 \hat{\psi}_{m}-150 \sin (0.9) t_{m}\right)\right)+\left(\hat{\psi}_{0}\right)^{2}, \\
& \varepsilon_{s-4, c-1}=0.1\left(\sum_{m=1}^{10}\left(\hat{\psi}_{m}^{\prime}+62.5 \hat{\psi}_{m}-150 \sin (0.3) t_{m}\right)^{2}\right)+\left(\hat{\psi}_{0}\right)^{2}
\end{aligned}
$$

The approximate solutions $\hat{\psi}(t)$ are obtained by using the optimized weights attained by GAsSQP algorithm for input interval $[0,1]$ with step size $h=0.05$ for both nonlinear RL problems. Reference solutions are also determined with the Adam numerical method for the same input grids. The magnitudes of performance operators MAE, ENSE and EVAF are determined for each independent trails. The best trail of the algorithm is identified on the basis of minimum magnitude of the indices as listed in Table 2 for each scenario of both nonlinear RL circuit problems.

The learning curves of GA and SQP, that is, iterative update of the fitness, are presented in the in Fig. 5 for the three cases. These learning curves show that the vary small improvement of the fitness by GAs with step increment in the generation, i.e., in steady state. The hybridization with local search algorithm by SQP enhance the steady state performance of GAs which is evident from subfigure 5(b), 5(d) and 5(f). The optimized parameter of NNDEMs for the runs of GASQP with MIN fitness for all three cases 1, 2 and 3 of scenario 1 and all four cases of scenarios 2 and 3 plotted in Fig. 6 in case of problem 1 based on DC excitation and accordingly for all cases of problem 2 based on AC excitation in Fig. A1 of appendix section.

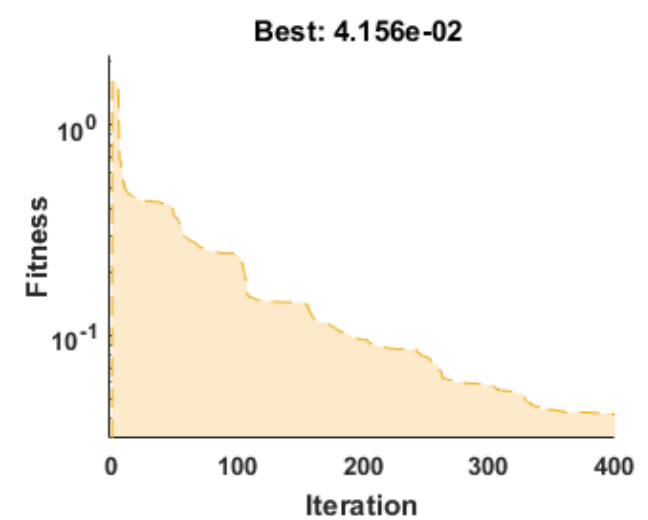

(a) LC of GAs for Scenario:1 Case:1

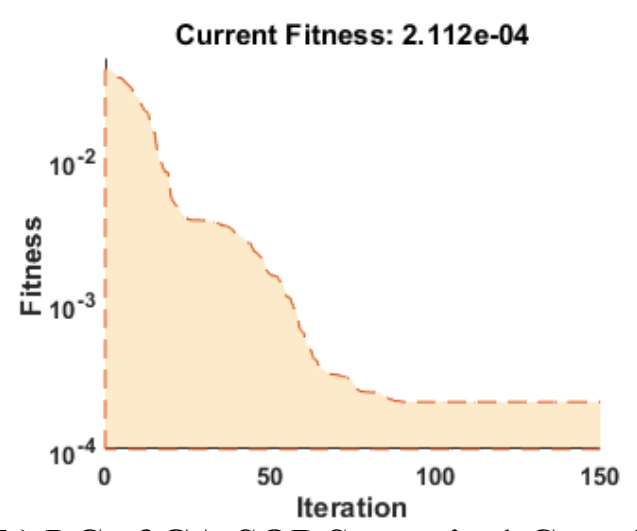

(b) LC of GA-SQP Scenario:1 Case:1 

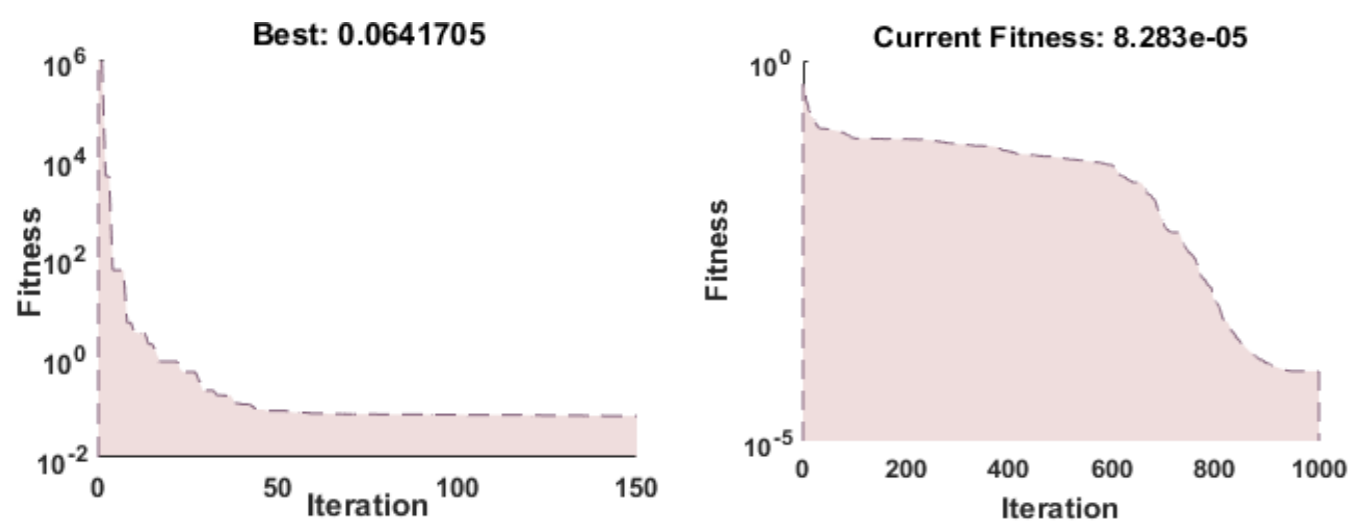

(c) LC of GA for Scenario:2 Case: 1

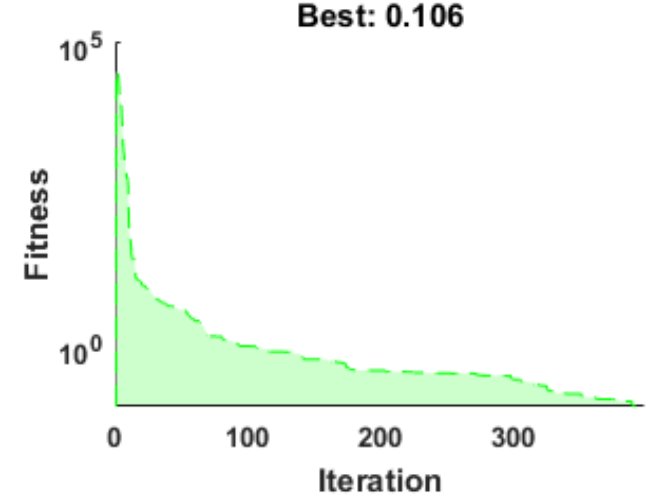

(d) LC of GA-SQP for Scenario:2 Case: 1 Current Fitness: $1.80 \mathrm{E}-4$

(e) LC of GA for Scenario:3 Case:1

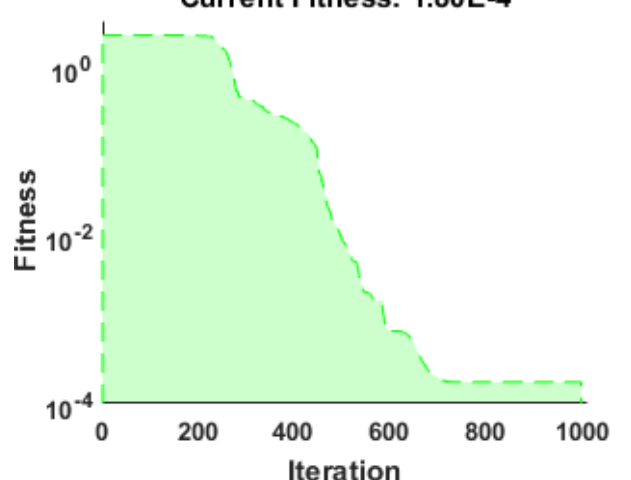

(f) LC of GA-SQP for Scenario:3 Case: 1

Fig. 5: Learning curves of optimization algorithms for training the weights of neural networks

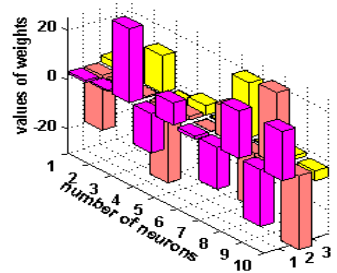

(a)Scenario-1, Case-1

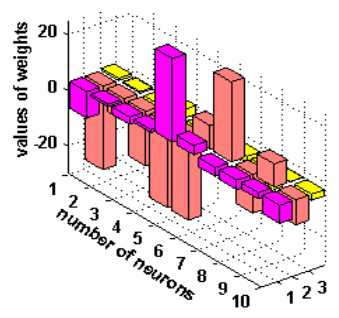

(e) Scenario-2, Case-2

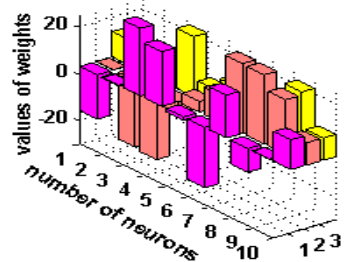

(b) Scenario-1, Case-2

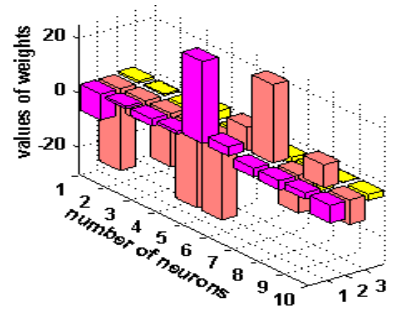

(f) Scenario-2, Case-3

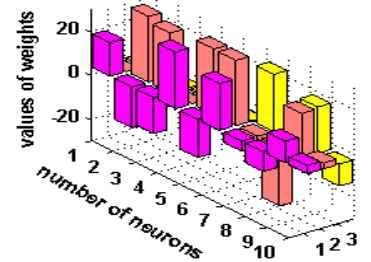

(c) Scenario-1, Case-3

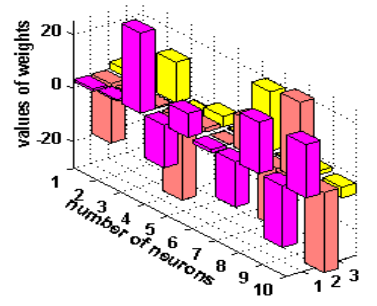

(g) Scenario-2, Case-4

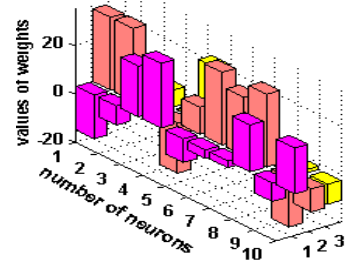

(d) Scenario-2, Case-1

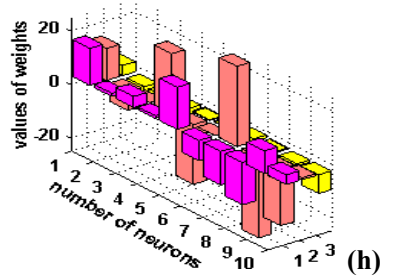

Scenario-3, Case-1 


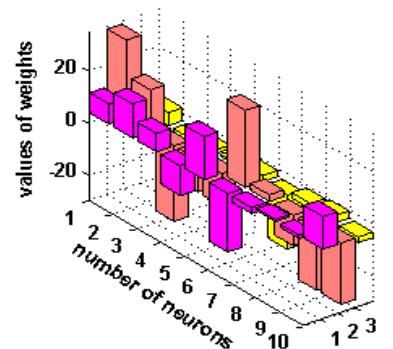

Scenario-3, Case-2

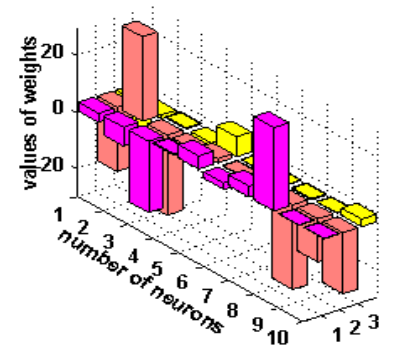

(i) Scenario-3, Case-3

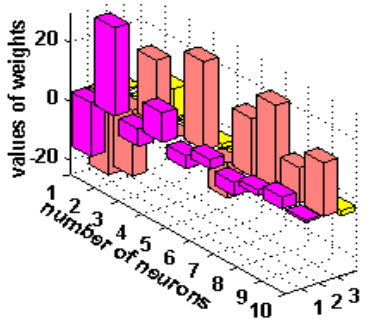

(j) Scenario-3, Case-4

Fig 6: Set of optimal weights of NNDEMs by GAs-SQP algorithm for dc excitation

The weights presented in Fig. 6 and Fig A1 of appendix are utilized to derive the proposed solutions for each scenario and are written below for case 1, for nonlinear RL circuit model for DC excitation as:

$$
\begin{aligned}
& \hat{\psi}_{s-1, c-1}(t)=\frac{9.3195}{1+e^{-(4.8409 t-0.1527)}}+\frac{25.0820}{1+e^{-(-29.7219 t-1.2781)}}+\ldots+\frac{-9.3540}{1+e^{-(26.1769 t-1.6330)}} \\
& \hat{\psi}_{s-2, c-1}(t)=\frac{-19.0746}{1+e^{-(0.1436 t+11.0013)}}+\frac{-0.4193}{1+e^{-(-28.5483 t-2.9034)}}+\ldots+\frac{12.7688}{1+e^{-(9.6196 t+9.6170)}} \\
& \hat{\psi}_{s-3, c-1}(t)=\frac{-18.6465}{1+e^{-(30.0000 t-1.43941)}}+\frac{-16.3230}{1+e^{-(27.3233 t-1.3456)}}+\ldots+\frac{3.6526}{1+e^{-(2.6116 t-10.0160)}}
\end{aligned}
$$

Similarly, the derived approximate solution for AC excitation for first case of each scenario is given as"

$$
\begin{aligned}
& \hat{\psi}_{s-1, c-1}(t)=\frac{0.8821}{1+e^{-(22.8641 t-6.7148)}}+\frac{0.2651}{1+e^{-(-20.1155 t-0.5350)}}+\ldots+\frac{-1.0771}{1+e^{-(22.5088 t+6.4738)}}, \\
& \hat{\psi}_{s-2-c-1}(t)=\frac{7.7529}{1+e^{-(29.9534 t-0.6773)}}+\frac{13.0596}{1+e^{-(16.0882 t+5.0939)}}+\ldots+\frac{12.5496}{1+e^{-(-23.6011 t-2.1449)}}, \\
& \hat{\psi}_{S-3, c-1}(t)=\frac{-3.2132}{1+e^{-(-22.76821 t-13.1075)}}+\frac{-6.6486}{1+e^{-(29.9078 t-1.39821)}}+\ldots+\frac{0.1680}{1+e^{-(-21.6583 t+2.6169)}}, \\
& \hat{\psi}_{S-4, c-1}(t)=\frac{-18.3658}{1+e^{-(-26.6085 t+0.6204)}}+\frac{30.0000}{1+e^{-(-22.5640 t-1.0500)}}+\ldots+\frac{0.7846}{1+e^{-(18.2508 t-1.6956)}},
\end{aligned}
$$

Table 2: The iteration of hybrid optimization GA-SQP with minimum (MIN) values of error based performance metrics for all three cases of each scenario.

\begin{tabular}{l|l|l|c|c|c|c|c|c}
\hline \hline \multirow{2}{*}{ Problem } & \multirow{2}{*}{ Scenario } & \multirow{2}{*}{ Index } & \multicolumn{2}{|c|}{ Case-1 } & \multicolumn{2}{c|}{ Case-2 } & \multicolumn{2}{c}{ Case-3 } \\
\cline { 4 - 7 } & & MIN & At Iteration & MIN & At Iteration & MIN & At Iteration \\
\hline \hline
\end{tabular}




\begin{tabular}{|c|c|c|c|c|c|c|c|c|}
\hline \multirow{9}{*}{1} & 1. & MAE & $1.03 \times 10^{-3}$ & 56 of 100 & $1.17 \times 10^{-3}$ & 51 of 100 & $4.88 \times 10^{-4}$ & 64 of 100 \\
\hline & & ENSE & $7.82 \times 10^{-6}$ & 19 of 100 & $7.79 \times 10^{-6}$ & 11 of 100 & $3.36 \times 10^{-6}$ & 64 of 100 \\
\hline & & EVAF & $6.61 \times 10^{-5}$ & 19 of 100 & $7.30 \times 10^{-5}$ & 79 of 100 & $2.63 \times 10^{-5}$ & 64 of 100 \\
\hline & 2. & MAE & $9.06 \times 10^{-4}$ & 55 of 100 & $7.59 \times 10^{-4}$ & 81 of 100 & $1.03 \times 10^{-3}$ & 56 of 100 \\
\hline & & ENSE & $1.60 \times 10^{-5}$ & 55 of 100 & $7.00 \times 10^{-6}$ & 75 of 100 & $7.82 \times 10^{-6}$ & 19 of 100 \\
\hline & & EVAF & $9.58 \times 10^{-5}$ & 55 of 100 & $5.22 \times 10^{-5}$ & 75 of 100 & $6.61 \times 10^{-5}$ & 19 of 100 \\
\hline & 3. & MAE & $6.53 \times 10^{-4}$ & 46 of 100 & $3.67 \times 10^{-4}$ & 83 of 100 & $3.12 \times 10^{-4}$ & 94 of 100 \\
\hline & & ENSE & $6.62 \times 10^{-6}$ & 70 of 100 & $5.04 \times 10^{-6}$ & 83 of 100 & $1.14 \times 10^{-6}$ & 94 of 100 \\
\hline & & EVAF & $4.35 \times 10^{-5}$ & 70 of 100 & $2.45 \times 10^{-5}$ & 83 of 100 & $1.91 \times 10^{-5}$ & 94 of 100 \\
\hline \multirow{12}{*}{2.} & 1. & MAE & $2.84 \times 10^{-3}$ & 35 of 100 & $3.74 \times 10^{-3}$ & 62 of 100 & $4.92 \times 10^{-3}$ & 87 of 100 \\
\hline & & ENSE & $3.22 \times 10^{-4}$ & 35 of 100 & $9.97 \times 10^{-4}$ & 62 of 100 & $4.78 \times 10^{-3}$ & 87 of 100 \\
\hline & & EVAF & $1.24 \times 10^{-3}$ & 35 of 100 & $1.59 \times 10^{-3}$ & 62 of 100 & $2.36 \times 10^{-3}$ & 87 of 100 \\
\hline & 2. & MAE & $1.53 \times 10^{-3}$ & 55 of 100 & $1.71 \times 10^{-3}$ & 51 of 100 & $4.56 \times 10^{-4}$ & 64 of 100 \\
\hline & & ENSE & $6.32 \times 10^{-6}$ & 17 of 100 & $6.49 \times 10^{-6}$ & 11 of 100 & $9.20 \times 10^{-6}$ & 64 of 100 \\
\hline & & EVAF & $6.34 \times 10^{-5}$ & 17 of 100 & $7.12 \times 10^{-5}$ & 79 of 100 & $3.46 \times 10^{-5}$ & 64 of 100 \\
\hline & 3. & MAE & $4.92 \times 10^{-3}$ & 87 of 100 & $1.31 \times 10^{-3}$ & 68 of 100 & $5.10 \times 10^{-4}$ & 64 of 100 \\
\hline & & ENSE & $4.78 \times 10^{-3}$ & 87 of 100 & $1.22 \times 10^{-4}$ & 95 of 100 & $1.84 \times 10^{-5}$ & 64 of 100 \\
\hline & & EVAF & $2.36 \times 10^{-3}$ & 87 of 100 & $6.18 \times 10^{-4}$ & 95 of 100 & $1.55 \times 10^{-4}$ & 64 of 100 \\
\hline & 4. & MAE & $2.04 \times 10^{-3}$ & 57 of 100 & $1.46 \times 10^{-3}$ & 49 of 100 & $8.84 \times 10^{-4}$ & 64 of 100 \\
\hline & & ENSE & $8.85 \times 10^{-6}$ & 18 of 100 & $5.54 \times 10^{-6}$ & 10 of 100 & $7.65 \times 10^{-6}$ & 64 of 100 \\
\hline & & EVAF & $4.62 \times 10^{-5}$ & 18 of 100 & $5.39 \times 10^{-5}$ & 77 of 100 & $3.62 \times 10^{-5}$ & 64 of 100 \\
\hline
\end{tabular}

Proposed approximate results of $\hat{\psi}(t)$ are obtained using weights provided in Fig. 6 and Fig. A1 of appendix while the calculated solutions are plotted in Figs. 7 and 8 for nonlinear RL problems 1 and 2, respectively, for each case. The results of Adam numerical solver (ANS) for the same grid points are also platted in Figs. 7 and 8 for problems 1 and 2, respectively. In order to access the level of matching between the approximate solutions and reference results, the values of absolute error (AE) are calculated and also plotted graphically in subfigures of both Figs. 7 and 8. In order to elaborate the minute difference in the results, the numerical values of $\mathrm{AE}$ are illustrated in Table 3 for DC excitation for nonlinear RL problems 1 for inputs between 0 and 1 with step size 0.05 , while these results for problem 2 are given in Table A1 of appendix. The presented results in show that the approximate solutions are regularly overlapping reference ANS for each case both problems and generally, the 2 to 7 decimal of accuracy is achieved for each variation of all three scenario of problem 1, while 3 to 8 decimal of accuracy attained for the variants of problem 2 .

Table 3: Comparative study on the basis of absolute error for the three cases of each scenario of problem 1 based on DC excitation 


\begin{tabular}{|c|c|c|c|c|c|c|c|c|}
\hline \multirow{2}{*}{ Scenario } & \multirow[b]{2}{*}{$\mathbf{t}$} & \multicolumn{3}{|c|}{ Absolute error } & \multirow[b]{2}{*}{$t$} & \multicolumn{3}{|c|}{ Absolute error } \\
\hline & & C: 1 & C: 2 & $C: 3$ & & C: 1 & C: 2 & $C: 3$ \\
\hline \multirow{10}{*}{1} & 0.05 & $9.22 \times 10^{-6}$ & $1.53 \times 10^{-2}$ & $1.91 \times 10^{-4}$ & 0.55 & $3.28 \times 10^{-5}$ & $1.33 \times 10^{-5}$ & $2.81 \times 10^{-5}$ \\
\hline & 0.10 & $1.94 \times 10^{-2}$ & $2.02 \times 10^{-2}$ & $9.33 \times 10^{-3}$ & 0.60 & $2.16 \times 10^{-5}$ & $5.41 \times 10^{-7}$ & $2.40 \times 10^{-5}$ \\
\hline & 0.15 & $1.75 \times 10^{-3}$ & $1.63 \times 10^{-3}$ & $4.50 \times 10^{-4}$ & 0.65 & $4.79 \times 10^{-6}$ & $1.02 \times 10^{-5}$ & $1.46 \times 10^{-5}$ \\
\hline & 0.20 & $2.04 \times 10^{-4}$ & $1.74 \times 10^{-4}$ & $3.69 \times 10^{-5}$ & 0.70 & $2.54 \times 10^{-5}$ & $1.93 \times 10^{-5}$ & $2.01 \times 10^{-6}$ \\
\hline & 0.25 & $3.03 \times 10^{-5}$ & $1.46 \times 10^{-4}$ & $5.06 \times 10^{-6}$ & 0.75 & $2.93 \times 10^{-5}$ & $2.71 \times 10^{-5}$ & $1.13 \times 10^{-5}$ \\
\hline & 0.30 & $1.24 \times 10^{-5}$ & $2.06 \times 10^{-4}$ & $3.13 \times 10^{-6}$ & 0.80 & $1.65 \times 10^{-5}$ & $3.38 \times 10^{-5}$ & $2.30 \times 10^{-5}$ \\
\hline & 0.35 & $4.15 \times 10^{-5}$ & $1.58 \times 10^{-4}$ & $3.01 \times 10^{-5}$ & 0.85 & $5.13 \times 10^{-6}$ & $3.95 \times 10^{-5}$ & $3.02 \times 10^{-5}$ \\
\hline & 0.40 & $2.82 \times 10^{-5}$ & $8.82 \times 10^{-5}$ & $1.46 \times 10^{-5}$ & 0.90 & $2.46 \times 10^{-5}$ & $4.46 \times 10^{-5}$ & $3.01 \times 10^{-5}$ \\
\hline & 0.45 & $2.93 \times 10^{-5}$ & $5.13 \times 10^{-5}$ & $9.6 \times 10^{-6}$ & 0.95 & $3.04 \times 10^{-5}$ & $4.90 \times 10^{-5}$ & $1.93 \times 10^{-5}$ \\
\hline & 0.50 & $1.13 \times 10^{-5}$ & $2.94 \times 10^{-5}$ & $2.44 \times 10^{-5}$ & 1.00 & $1.23 \times 10^{-5}$ & $5.29 \times 10^{-5}$ & $6.15 \times 10^{-6}$ \\
\hline \multirow{10}{*}{2} & 0.05 & $5.77 \times 10^{-4}$ & $1.36 \times 10^{-4}$ & $9.25 \times 10^{-6}$ & 0.55 & $1.25 \times 10^{-5}$ & $6.42 \times 10^{-6}$ & $3.28 \times 10^{-5}$ \\
\hline & 0.10 & $1.52 \times 10^{-3}$ & $1.36 \times 10^{-2}$ & $1.93 \times 10^{-2}$ & 0.60 & $9.25 \times 10^{-5}$ & $1.56 \times 10^{-5}$ & $2.16 \times 10^{-5}$ \\
\hline & 0.15 & $1.41 \times 10^{-3}$ & $1.12 \times 10^{-3}$ & $1.74 \times 10^{-3}$ & 0.65 & $3.89 \times 10^{-5}$ & $2.45 \times 10^{-5}$ & $4.79 \times 10^{-6}$ \\
\hline & 0.20 & $2.36 \times 10^{-4}$ & $1.55 \times 10^{-4}$ & $2.00 \times 10^{-4}$ & 0.70 & $1.80 \times 10^{-5}$ & $2.71 \times 10^{-5}$ & $2.54 \times 10^{-5}$ \\
\hline & 0.25 & $5.42 \times 10^{-5}$ & $6.99 \times 10^{-5}$ & $3.01 \times 10^{-5}$ & 0.75 & $6.31 \times 10^{-5}$ & $2.71 \times 10^{-5}$ & $2.93 \times 10^{-5}$ \\
\hline & 0.30 & $2.33 \times 10^{-4}$ & $1.94 \times 10^{-4}$ & $1.22 \times 10^{-5}$ & 0.80 & $8.86 \times 10^{-5}$ & $2.63 \times 10^{-5}$ & $1.65 \times 10^{-5}$ \\
\hline & 0.35 & $1.02 \times 10^{-4}$ & $4.76 \times 10^{-5}$ & $4.07 \times 10^{-5}$ & 0.85 & $9.09 \times 10^{-5}$ & $2.55 \times 10^{-5}$ & $5.13 \times 10^{-6}$ \\
\hline & 0.40 & $1.53 \times 10^{-4}$ & $1.46 \times 10^{-4}$ & $2.80 \times 10^{-5}$ & 0.90 & $6.91 \times 10^{-5}$ & $2.48 \times 10^{-5}$ & $2.46 \times 10^{-5}$ \\
\hline & 0.45 & $2.57 \times 10^{-5}$ & $1.10 \times 10^{-4}$ & $2.92 \times 10^{-5}$ & 0.95 & $2.31 \times 10^{-5}$ & $2.43 \times 10^{-5}$ & $3.04 \times 10^{-5}$ \\
\hline & 0.50 & $8.39 \times 10^{-5}$ & $4.92 \times 10^{-5}$ & $1.13 \times 10^{-5}$ & 1.00 & $4.67 \times 10^{-5}$ & $2.40 \times 10^{-5}$ & $1.23 \times 10^{-5}$ \\
\hline \multirow{9}{*}{3} & 0.05 & $7.17 \times 10^{-4}$ & $8.94 \times 10^{-5}$ & $4.75 \times 10^{-4}$ & 0.55 & $3.68 \times 10^{-6}$ & $1.27 \times 10^{-5}$ & $1.61 \times 10^{-5}$ \\
\hline & 0.15 & $1.16 \times 10^{-3}$ & $3.83 \times 10^{-4}$ & $3.72 \times 10^{-4}$ & 0.65 & $1.12 \times 10^{-5}$ & $1.04 \times 10^{-5}$ & $3.44 \times 10^{-5}$ \\
\hline & 0.20 & $1.76 \times 10^{-4}$ & $3.24 \times 10^{-5}$ & $3.89 \times 10^{-5}$ & 0.70 & $6.97 \times 10^{-6}$ & $4.20 \times 10^{-6}$ & $3.04 \times 10^{-5}$ \\
\hline & 0.25 & $1.90 \times 10^{-5}$ & $7.15 \times 10^{-6}$ & $6.46 \times 10^{-5}$ & 0.75 & $2.74 \times 10^{-6}$ & $2.31 \times 10^{-6}$ & $2.33 \times 10^{-5}$ \\
\hline & 0.30 & $6.07 \times 10^{-5}$ & $2.58 \times 10^{-5}$ & $1.29 \times 10^{-4}$ & 0.80 & $2.48 \times 10^{-7}$ & $7.59 \times 10^{-6}$ & $1.50 \times 10^{-5}$ \\
\hline & 0.35 & $7.31 \times 10^{-5}$ & $9.35 \times 10^{-6}$ & $7.56 \times 10^{-6}$ & 0.85 & $1.96 \times 10^{-6}$ & $1.06 \times 10^{-5}$ & $6.44 \times 10^{-6}$ \\
\hline & 0.40 & $3.35 \times 10^{-6}$ & $2.00 \times 10^{-5}$ & $8.26 \times 10^{-5}$ & 0.90 & $2.74 \times 10^{-6}$ & $1.04 \times 10^{-5}$ & $2.12 \times 10^{-6}$ \\
\hline & 0.45 & $3.54 \times 10^{-5}$ & $9.82 \times 10^{-6}$ & $6.25 \times 10^{-5}$ & 0.95 & $2.97 \times 10^{-6}$ & $6.46 \times 10^{-6}$ & $1.06 \times 10^{-5}$ \\
\hline & 0.50 & $1.72 \times 10^{-5}$ & $4.18 \times 10^{-6}$ & $1.72 \times 10^{-5}$ & 1.00 & $2.91 \times 10^{-6}$ & $1.80 \times 10^{-6}$ & $1.90 \times 10^{-5}$ \\
\hline
\end{tabular}




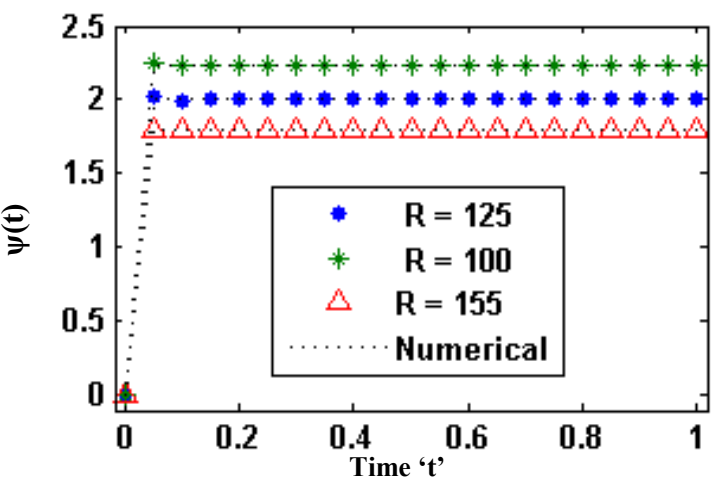

(a)Scenario 1

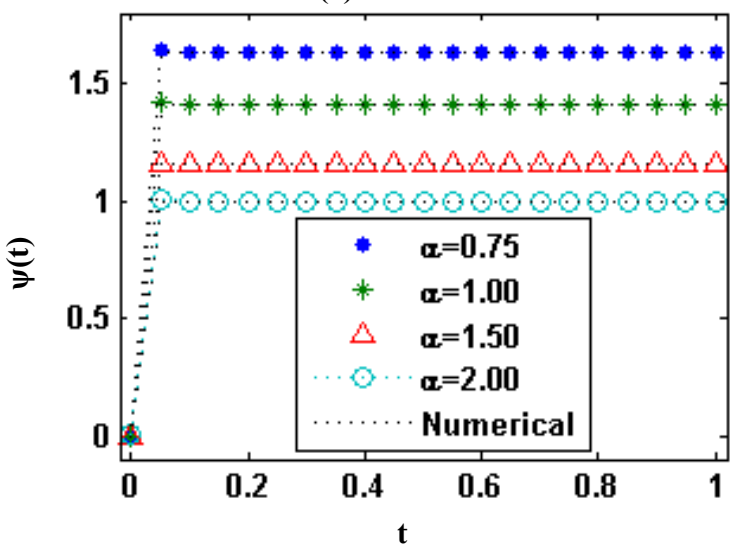

(c) Scenario 3

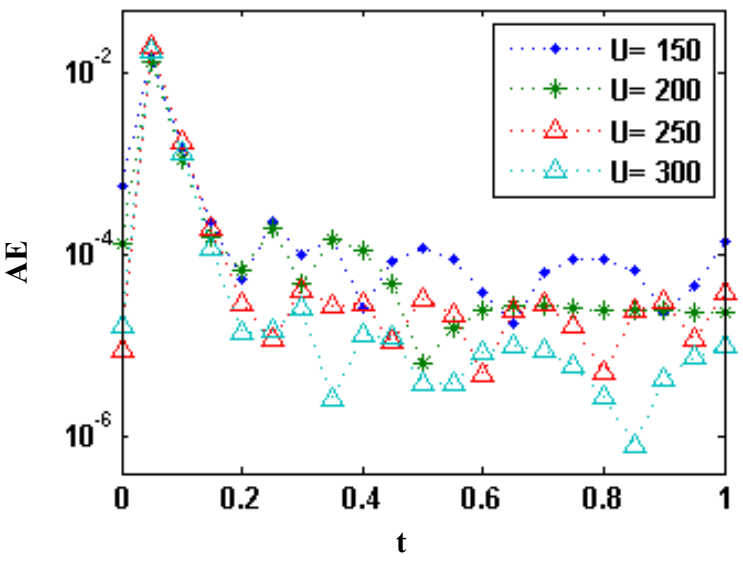

(e) Scenario 2

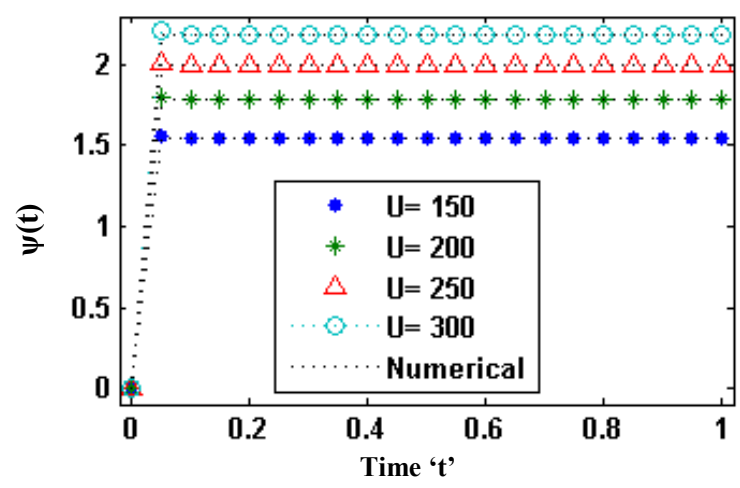

(b) Scenario 2

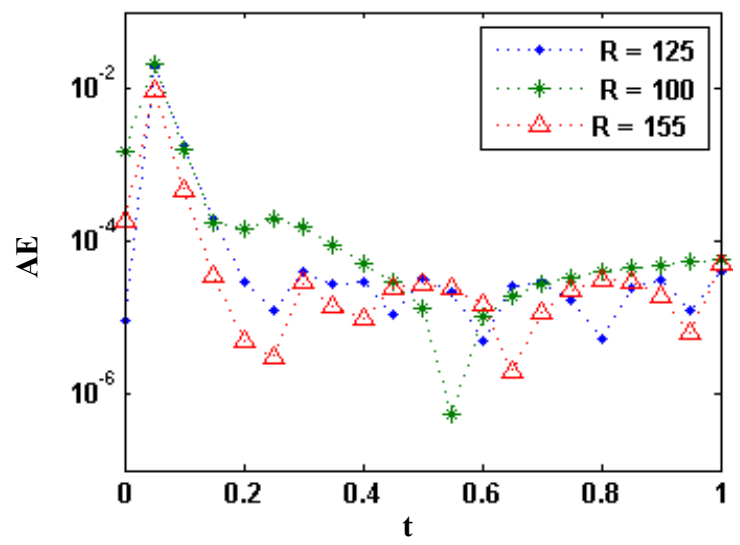

(d) Scenario 1

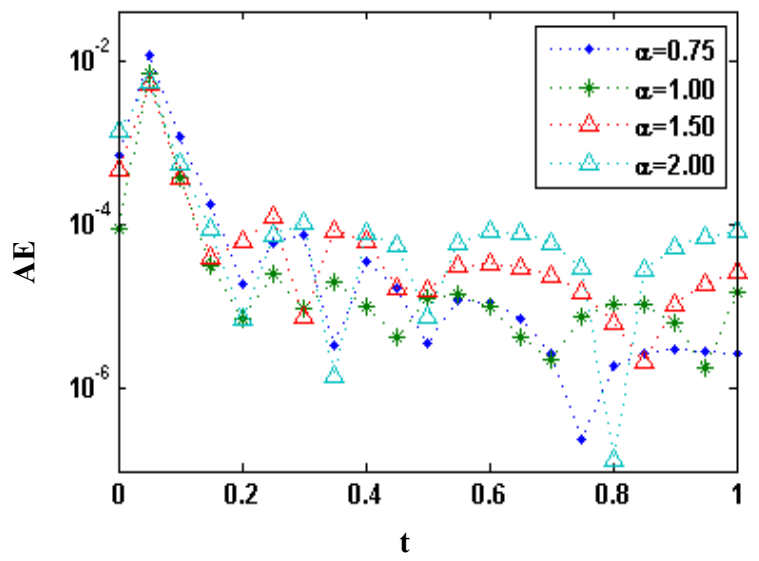

(f) Scenario 3

Fig. 7: Comparison of proposed solutions for various nonlinear RL Circuits models for DC excitation 


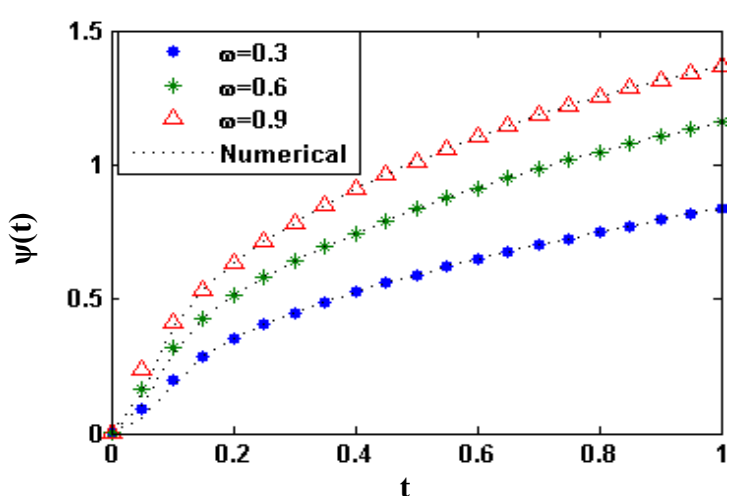

(a) Scenario 1

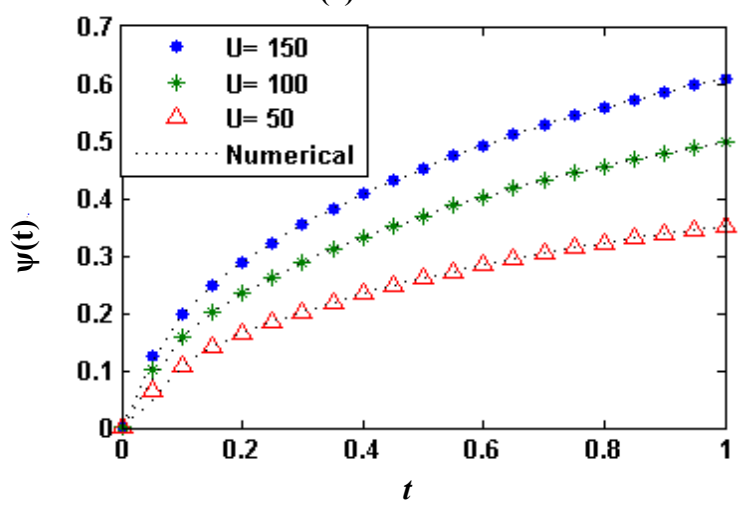

(c) Scenario 3

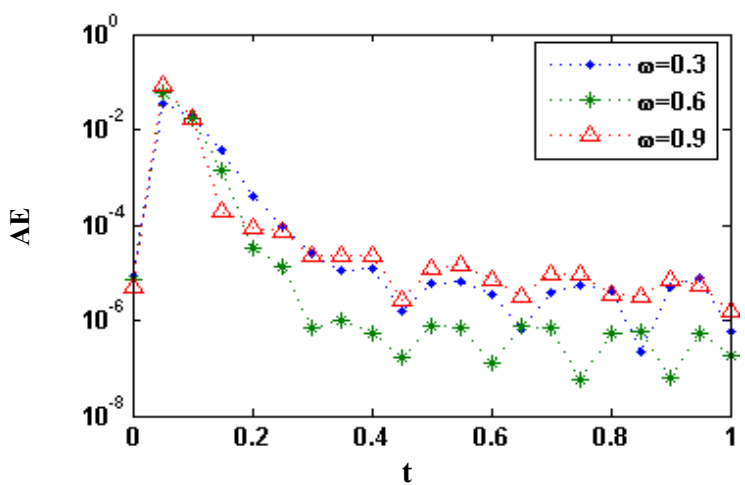

(e) Scenario 1

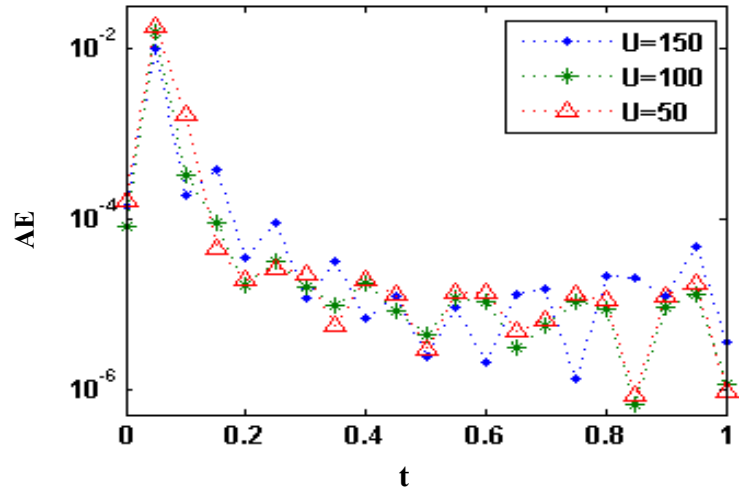

(g) Scenario 3

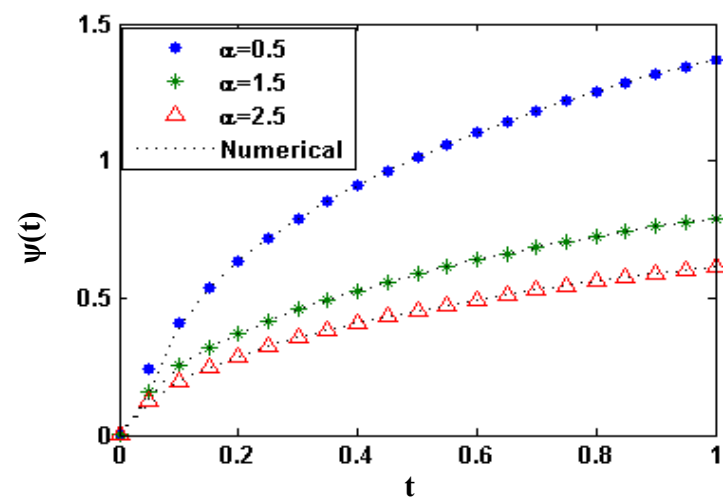

(b) Scenario 2

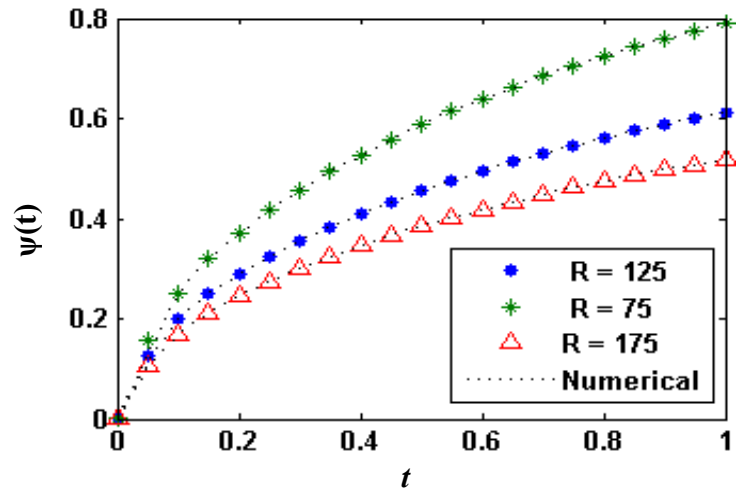

(d) Scenario: 4

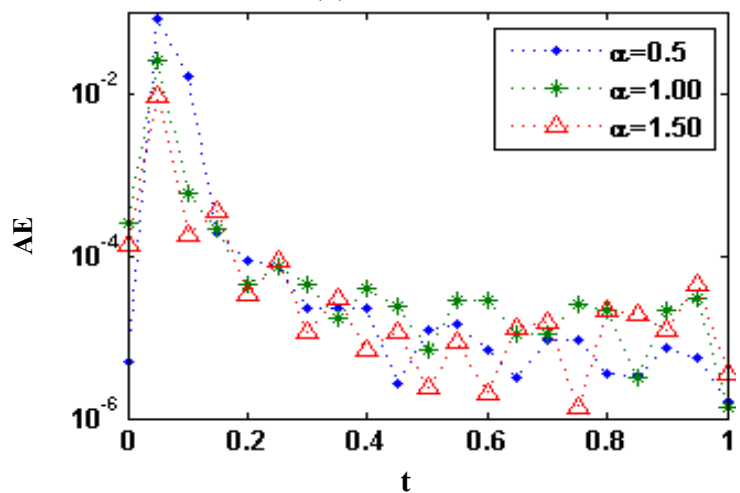

(f) Scenario 2

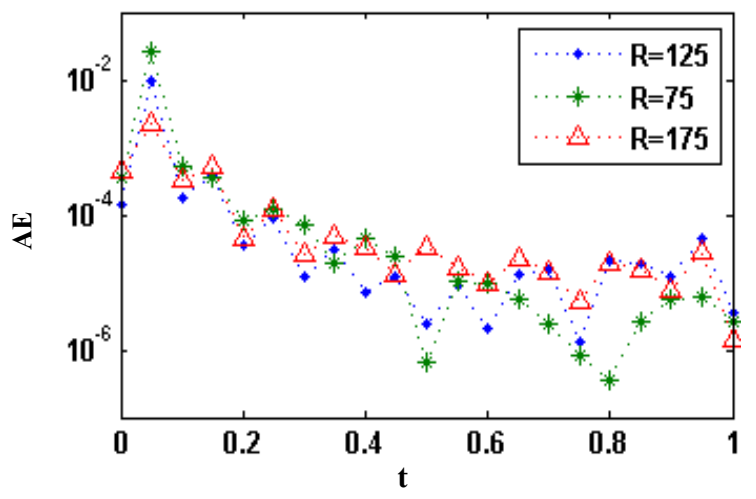

(h) Scenario 4

Fig. 8: Proposed solutions for various nonlinear RL Circuits models for AC excitation 


\section{Comparative Studies through Statistical Analyses}

Multiple runs of the proposed NNDEM optimized with GA-SQP are performed to access the effective performance of the scheme for solving nonlinear RL circuit model. The statistical analysis for 100 trails of the design scheme is conducted both DC and AC excitation of nonlinear RL circuit system and necessary description is provided in this section.

Performance of the heuristic technique, NNDEM optimized with GA-SQP, is examined by mean of MAE, VAF and NSE operators for 100 multiple runs. Results in terms of MAE magnitudes for multiple independent runs are plotted in unsorted semi-log graphs in Figs. 9 and 10 for DC and AC excitation based RL circuit model, respectively. Similarly, respective results of NSE and VAF both for problem 1 and problem 2 are shown in Figs. A2 to A5 of the appendix section. It is observed that very low magnitudes of these performance indicators MAE, ENSE, and EVAF are attained consistently, which demonstrate the invariable accuracy of the scheme.
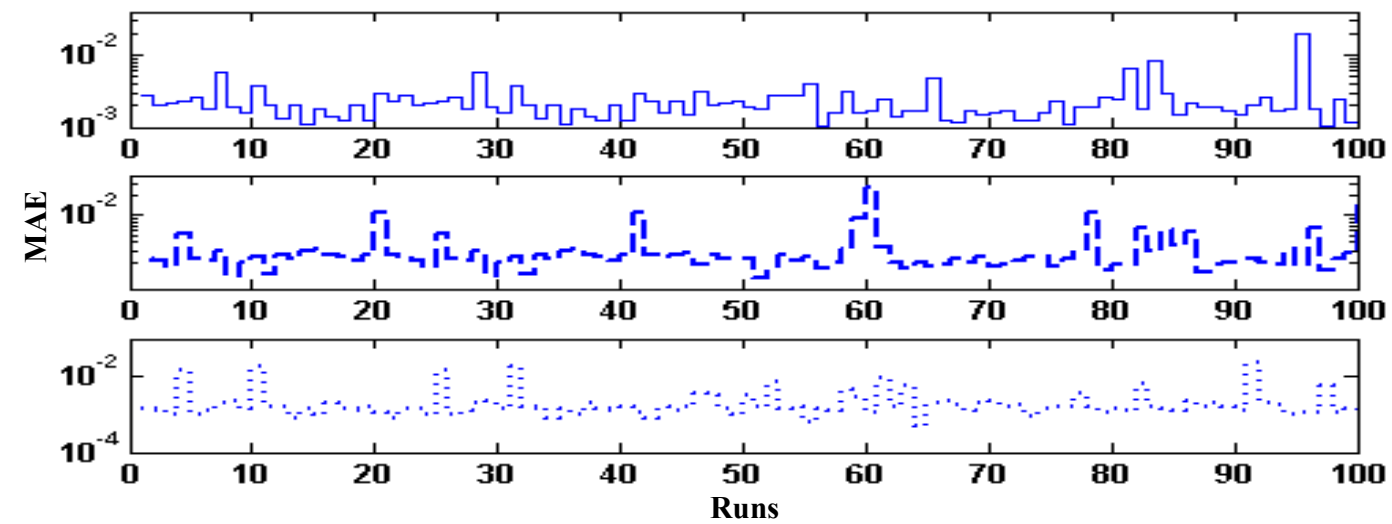

Scenario 1; DC Excitation

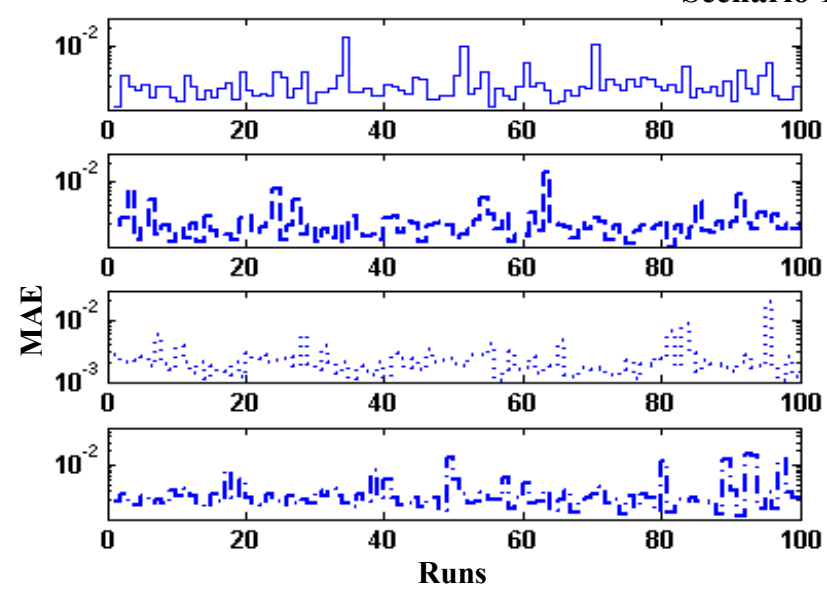

Scenario 2; DC Excitation
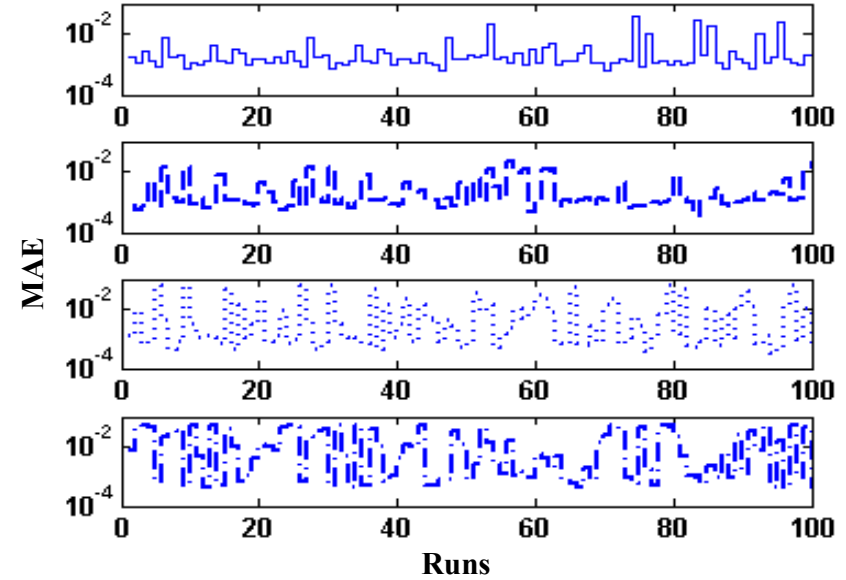

Scenario 3; DC Excitation

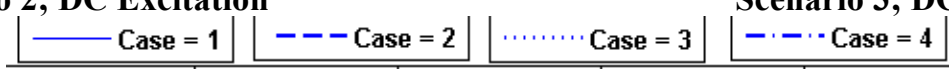

Fig. 9: Comparison on MAE operator for multiple runs of the algorithm for each variant of nonlinear RL circuit considering DC excitation 

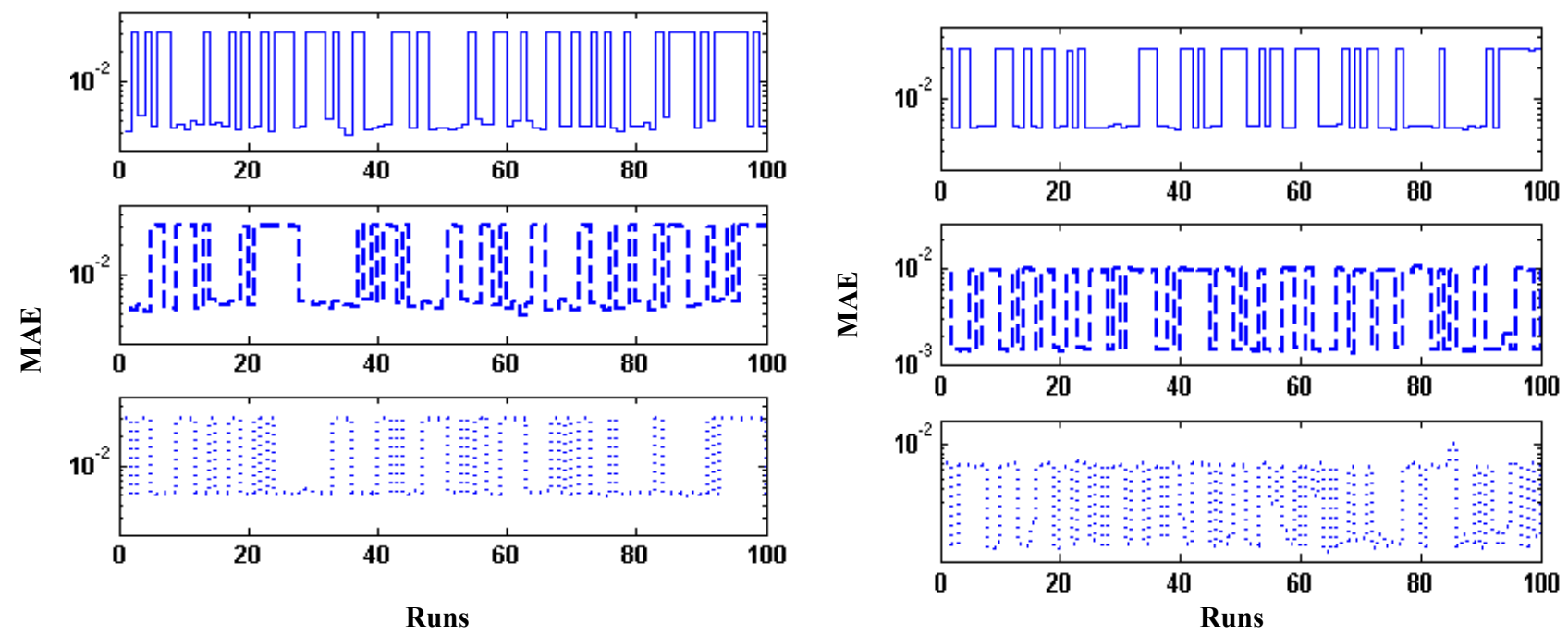

Scenario 1; AC Excitation
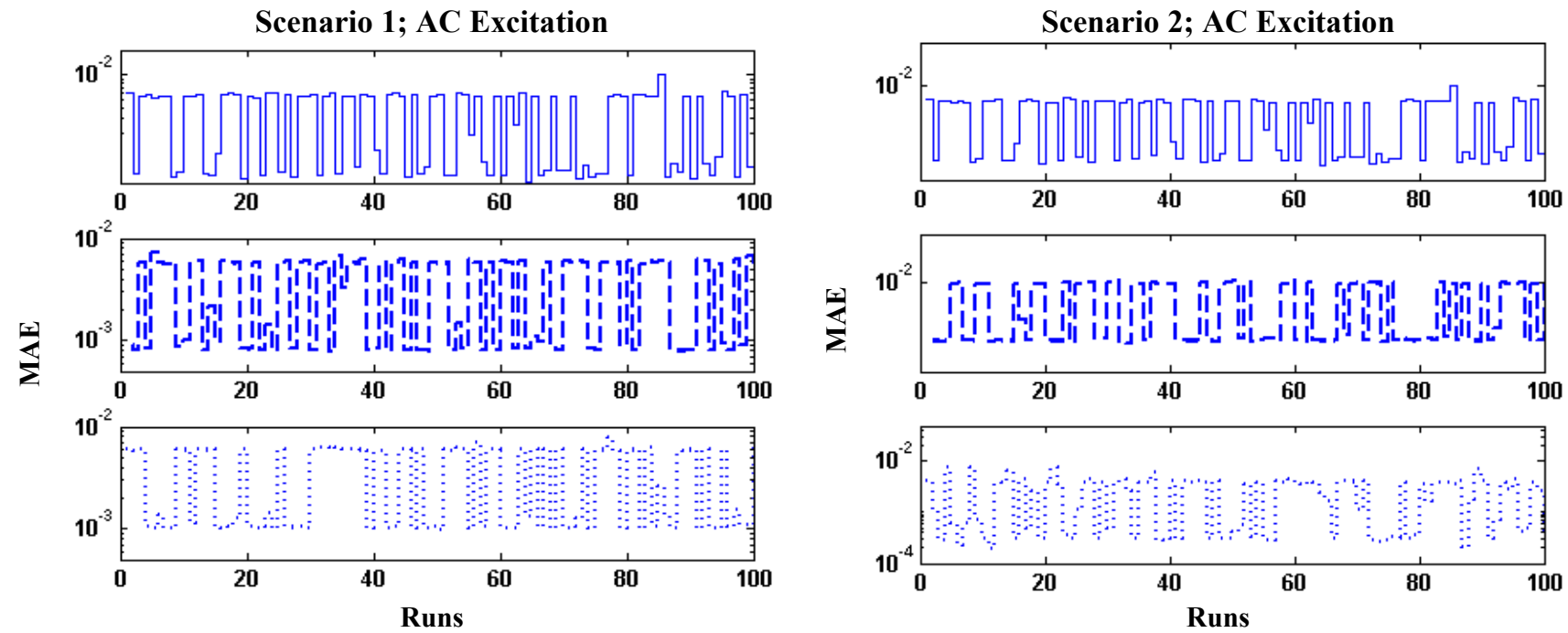

Scenario 3; AC Excitation

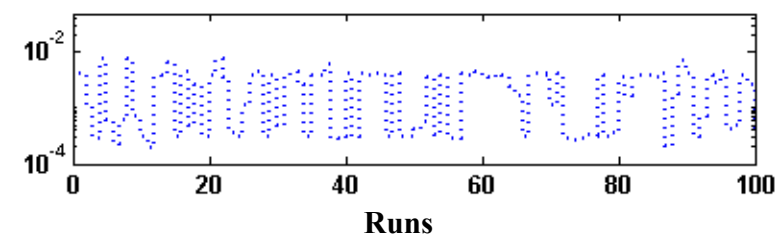

Scenario 4; AC Excitation

$\left\lfloor\begin{array}{l|l|l|l|l|}- \text { Case }=1 \\ \hline\end{array}\right.$

Fig. 10: Comparison on MAE operator for multiple runs of the algorithm for each variant of nonlinear RL circuit considering AC excitation

The accuracy and convergence of the proposed hybrid strategy is investigated further in terms of statistical indices of mean, standard deviation (STD) and minimum (MIN) gauges of the AE. The results of statistics on the basis of 100 independent trials are tabulated in Table 4 and Table A2 of appendix section for inputs $x \in(0,1)$ with step size of 0.2 for all cases of nonlinear RL circuit in case of DC and AC excitation, respectively. It is seen that the values lie in the range of $10^{-02}$ to $10^{-07}$ and $10^{-03}$ to $10^{-08}$ for problem-1 and problem 2, respectively. Generally, it is stated that results are accurate, having smaller values of the statistical indices, that prove consistency in precision and convergence of the proposed algorithm. 
Further evaluation of efficacy of the proposed algorithm is performed through percentage convergent runs by attaining the various accuracy stages for fitness and MAE, i.e., $\varepsilon \leq\left(10^{-04}, 10^{-}\right.$ ${ }^{05}, 10^{-07}$ and $\left.10^{-09}\right)$, and MAE $\leq\left(10^{-03}, 10^{-04}, 10^{-05}\right.$ and $\left.10^{-06}\right)$. Results in terms of percentage trials fulfilling these accuracy measures are given in Table 5 which show that around $90+\%$ of the independent trials proposed methodology based on NNDEM optimized with GA-SQP remains convergent on base criterion of both performance measures while few independent trial attain the level of relatively tough criteria.

Comparative analysis on the performance of the scheme is continues by means of global indicators of GMAE, $\mathrm{EG}_{\mathrm{NSE}}$, and $\mathrm{EG}_{\mathrm{VAF}}$ on the basis of 100 independent trials. Magnitudes of these global indices for each case of nonlinear RL circuit problem 1 with DC, as well as, problem 2 with AC excitation are listed in Table 6. The presented results with very small magnitudes of global metrics established the invariable consistency, stability and robustness of the design methodology to solve each case of the scenarios of nonlinear RL circuit model given in problem 1 and 2.

\section{Conclusions}

Neurocomputing paradigm is designed effectively for solving RL circuit models having nonlinear inductance excited with both DC and AC source voltage by exploiting the potential of accurate artificial neural network modeling optimized globally with genetic algorithms hybrid with sequential quadratic programming for viable local search. The designed solver is applied to various scenarios of nonlinear RL circuit problems with DC as well as AC excitation based on different magnitudes of resistance, inductance, and voltage parameters. Results reveal that approximate solutions consistently matched with the Adams numerical method within a range of 3 to 8 decimal places of accuracy for each variant of RL circuit models. Comparison through statistical analysis based on 100 independent trials in terms of mean and standard deviation verify the consistency and convergence of the designed scheme for each nonlinear circuit problem. Comparative study of the proposed scheme is further validated through performance metrics i.e., mean of absolute error, variance account for and Nash-Sutcliffe efficiency and their global versions for 100 independent trials of the algorithms. Smaller values of these operators demonstrate the accuracy and efficacy of the designed scheme. Beside high prescient and reliability the implementation ease, simple conceptual procedures, broader applicability and extendibility are other hallmarks of the designed methodology.

In future, it looks promising to exploit the proposed methodology solving the problems arising in nonlinear circuit analysis based on continuous values of the voltages. Additionally, the proposed scheme is a good alternative to be exploited for stiff optimization problems based on flexoelectric materials [65-66]. 
Table 4: Results based on statistical operators for different variants of nonlinear RL circuits for dc excitation

\begin{tabular}{|c|c|c|c|c|c|c|c|}
\hline \multirow{2}{*}{ Scenario } & \multirow{2}{*}{ Case } & \multirow{2}{*}{ Model } & \multicolumn{5}{|c|}{ Absolute Error for input ' $t$ ' } \\
\hline & & & 0.1 & 0.3 & 0.5 & 0.7 & 0.9 \\
\hline \multirow{9}{*}{1} & \multirow{3}{*}{1} & $\overline{\mathrm{MIN}}$ & $9.25 \times 10^{-6}$ & $1.65 \times 10^{-6}$ & $1.45 \times 10^{-6}$ & $3.25 \times 10^{-7}$ & $1.94 \times 10^{-6}$ \\
\hline & & Mean & $1.35 \times 10^{-2}$ & $3.37 \times 10^{-4}$ & $1.20 \times 10^{-4}$ & $7.79 \times 10^{-5}$ & $1.33 \times 10^{-4}$ \\
\hline & & STD & 4.15E-02 & $3.29 \times 10^{-4}$ & $1.37 \times 10^{-4}$ & $7.54 \times 10^{-5}$ & $1.62 \times 10^{-4}$ \\
\hline & \multirow{3}{*}{2} & MIN & $2.88 \times 10^{-5}$ & $4.96 \times 10^{-6}$ & $5.09 \times 10^{-7}$ & $5.04 \times 10^{-7}$ & $9.47 \times 10^{-7}$ \\
\hline & & Mean & $2.21 \times 10^{-2}$ & $4.66 \times 10^{-4}$ & $1.48 \times 10^{-4}$ & $9.71 \times 10^{-5}$ & $1.71 \times 10^{-4}$ \\
\hline & & STD & $6.50 \times 10^{-2}$ & $6.36 \times 10^{-4}$ & $2.02 \times 10^{-4}$ & $1.52 \times 10^{-4}$ & $2.36 \times 10^{-4}$ \\
\hline & \multirow{3}{*}{3} & MIN & $1.91 \times 10^{-5}$ & $3.06 \times 10^{-6}$ & $1.80 \times 10^{-6}$ & $2.20 \times 10^{-8}$ & $9.05 \times 10^{-7}$ \\
\hline & & Mean & $2.79 \times 10^{-2}$ & $3.99 \times 10^{-4}$ & $1.33 \times 10^{-4}$ & $1.07 \times 10^{-4}$ & $1.95 \times 10^{-4}$ \\
\hline & & STD & $7.56 \times 10^{-2}$ & $4.80 \times 10^{-4}$ & $1.98 \times 10^{-4}$ & $1.56 \times 10^{-4}$ & $3.50 \times 10^{-4}$ \\
\hline \multirow{12}{*}{2} & \multirow{3}{*}{1} & MIN & $9.45 \times 10^{-7}$ & $2.00 \times 10^{-5}$ & $9.82 \times 10^{-7}$ & $7.09 \times 10^{-8}$ & $1.09 \times 10^{-6}$ \\
\hline & & Mean & $1.22 \times 10^{-2}$ & $3.55 \times 10^{-4}$ & $1.28 \times 10^{-4}$ & $9.56 \times 10^{-5}$ & $1.37 \times 10^{-4}$ \\
\hline & & STD & $3.32 \times 10^{-2}$ & $4.24 \times 10^{-4}$ & $1.68 \times 10^{-4}$ & $1.25 \times 10^{-4}$ & $1.47 \times 10^{-4}$ \\
\hline & \multirow{3}{*}{2} & MIN & $1.67 \times 10^{-5}$ & $1.04 \times 10^{-5}$ & $3.74 \times 10^{-6}$ & $7.97 \times 10^{-7}$ & $2.02 \times 10^{-6}$ \\
\hline & & Mean & $2.61 \times 10^{-2}$ & $4.41 \times 10^{-4}$ & $1.41 \times 10^{-4}$ & $8.17 \times 10^{-5}$ & $1.53 \times 10^{-4}$ \\
\hline & & STD & $6.25 \times 10^{-2}$ & $6.17 \times 10^{-4}$ & $1.90 \times 10^{-4}$ & $1.11 \times 10^{-4}$ & $2.66 \times 10^{-4}$ \\
\hline & \multirow{3}{*}{3} & MIN & $9.25 \times 10^{-6}$ & $1.65 \times 10^{-6}$ & $1.45 \times 10^{-6}$ & $3.25 \times 10^{-7}$ & $1.94 \times 10^{-6}$ \\
\hline & & Mean & $1.35 \times 10^{-2}$ & $3.37 \times 10^{-4}$ & $1.20 \times 10^{-4}$ & $7.79 \times 10^{-5}$ & $1.33 \times 10^{-4}$ \\
\hline & & STD & $4.15 \mathrm{E}-02$ & $3.29 \times 10^{-4}$ & $1.37 \times 10^{-4}$ & $7.54 \times 10^{-5}$ & $1.62 \times 10^{-4}$ \\
\hline & \multirow{3}{*}{4} & MIN & $4.52 \times 10^{-5}$ & $5.14 \times 10^{-6}$ & $3.06 \times 10^{-6}$ & $3.86 \times 10^{-7}$ & $1.50 \times 10^{-6}$ \\
\hline & & Mean & $1.72 \times 10^{-2}$ & $4.85 \times 10^{-4}$ & $1.84 \times 10^{-4}$ & $1.22 \times 10^{-4}$ & $1.99 \times 10^{-4}$ \\
\hline & & STD & $4.46 \times 10^{-2}$ & $5.24 \times 10^{-4}$ & $2.07 \times 10^{-4}$ & $1.31 \times 10^{-4}$ & $2.25 \times 10^{-4}$ \\
\hline \multirow{12}{*}{3} & \multirow{3}{*}{1} & MIN & $9.66 \times 10^{-5}$ & $3.31 \times 10^{-6}$ & $1.14 \times 10^{-6}$ & $1.12 \times 10^{-7}$ & $1.18 \times 10^{-6}$ \\
\hline & & Mean & $3.18 \times 10^{-1}$ & $3.20 \times 10^{-4}$ & $1.12 \times 10^{-4}$ & $1.01 \times 10^{-4}$ & $1.58 \times 10^{-4}$ \\
\hline & & STD & $3.91 \times 10^{-1}$ & $3.15 \times 10^{-4}$ & $1.41 \times 10^{-4}$ & $1.49 \times 10^{-4}$ & $1.99 \times 10^{-4}$ \\
\hline & \multirow{3}{*}{2} & MIN & $6.43 \times 10^{-6}$ & $4.60 \times 10^{-6}$ & $3.68 \times 10^{-6}$ & $2.48 \times 10^{-7}$ & $1.66 \times 10^{-7}$ \\
\hline & & Mean & $4.48 \times 10^{-2}$ & $4.37 \times 10^{-4}$ & $1.70 \times 10^{-4}$ & $1.33 \times 10^{-4}$ & $2.00 \times 10^{-4}$ \\
\hline & & STD & $1.24 \times 10^{-1}$ & $4.38 \times 10^{-4}$ & $2.16 \times 10^{-4}$ & $1.71 \times 10^{-4}$ & $1.84 \times 10^{-4}$ \\
\hline & \multirow{3}{*}{3} & MIN & $2.16 \times 10^{-6}$ & $5.54 \times 10^{-6}$ & $9.05 \times 10^{-7}$ & $5.31 \times 10^{-7}$ & $1.30 \times 10^{-6}$ \\
\hline & & Mean & $4.81 \times 10^{-2}$ & $4.14 \times 10^{-4}$ & $1.33 \times 10^{-4}$ & $1.17 \times 10^{-4}$ & $1.85 \times 10^{-4}$ \\
\hline & & STD & $9.36 \times 10^{-2}$ & $4.30 \times 10^{-4}$ & $1.53 \times 10^{-4}$ & $1.34 \times 10^{-4}$ & $2.14 \times 10^{-4}$ \\
\hline & \multirow{3}{*}{4} & MIN & $1.90 \times 10^{-1}$ & $3.72 \times 10^{-4}$ & $1.59 \times 10^{-4}$ & $1.24 \times 10^{-4}$ & $2.46 \times 10^{-4}$ \\
\hline & & Mean & $3.29 \times 10^{-1}$ & $3.71 \times 10^{-4}$ & $1.85 \times 10^{-4}$ & $1.53 \times 10^{-4}$ & $3.11 \times 10^{-4}$ \\
\hline & & STD & $1.23 \times 10^{-1}$ & $2.67 \times 10^{-4}$ & $1.57 \times 10^{-4}$ & $1.25 \times 10^{-4}$ & $1.87 \times 10^{-4}$ \\
\hline
\end{tabular}

Table 5: Percentage convergence for various nonlinear RL circuit Models 


\begin{tabular}{|c|c|c|c|c|c|c|c|c|c|c|}
\hline \multirow{3}{*}{ Problem } & \multirow{3}{*}{ Scenario } & \multirow{3}{*}{ Case } & \multicolumn{8}{|c|}{ Performance Measures } \\
\hline & & & \multicolumn{4}{|c|}{$\%$ runs with fitness } & \multicolumn{4}{|c|}{$\%$ runs with MAE } \\
\hline & & & $10^{-04}$ & $10^{-05}$ & $10^{-07}$ & $10^{-09}$ & $10^{-03}$ & $10^{-04}$ & $10^{-05}$ & $10^{-06}$ \\
\hline \multirow{11}{*}{1} & \multirow{3}{*}{1} & 1 & 94 & 34 & 2 & 0 & 100 & 100 & 95 & 0 \\
\hline & & 2 & 91 & 37 & 5 & 1 & 100 & 100 & 88 & 0 \\
\hline & & 3 & 89 & 26 & 5 & 0 & 100 & 100 & 90 & 1 \\
\hline & \multirow{4}{*}{2} & 1 & 96 & 37 & 8 & 0 & 100 & 100 & 95 & 0 \\
\hline & & 2 & 94 & 34 & 2 & 0 & 100 & 100 & 95 & 0 \\
\hline & & 3 & 95 & 34 & 4 & 1 & 100 & 100 & 90 & 0 \\
\hline & & 4 & 95 & 34 & 4 & 1 & 100 & 100 & 95 & 0 \\
\hline & \multirow{4}{*}{3} & 1 & 52 & 11 & 1 & 1 & 100 & 100 & 51 & 10 \\
\hline & & 2 & 90 & 19 & 4 & 0 & 100 & 100 & 90 & 0 \\
\hline & & 3 & 85 & 21 & 8 & 0 & 100 & 100 & 83 & 2 \\
\hline & & 4 & 68 & 13 & 1 & 0 & 100 & 94 & 64 & 9 \\
\hline \multirow{12}{*}{2} & \multirow{3}{*}{1} & 1 & 100 & 94 & 80 & 58 & 100 & 100 & 56 & 0 \\
\hline & & 2 & 100 & 98 & 61 & 38 & 100 & 100 & 31 & 0 \\
\hline & & 3 & 100 & 93 & 66 & 15 & 100 & 100 & 3 & 0 \\
\hline & \multirow{3}{*}{2} & 1 & 100 & 84 & 47 & 17 & 100 & 100 & 44 & 0 \\
\hline & & 2 & 100 & 98 & 61 & 38 & 100 & 100 & 31 & 0 \\
\hline & & 3 & 100 & 87 & 38 & 10 & 100 & 100 & 45 & 0 \\
\hline & \multirow{3}{*}{3} & 1 & 100 & 94 & 80 & 58 & 100 & 100 & 56 & 0 \\
\hline & & 2 & 100 & 89 & 51 & 16 & 100 & 100 & 46 & 0 \\
\hline & & 3 & 100 & 95 & 64 & 34 & 100 & 100 & 51 & 0 \\
\hline & \multirow{3}{*}{4} & 1 & 100 & 85 & 62 & 19 & 100 & 100 & 57 & 0 \\
\hline & & 2 & 100 & 79 & 40 & 12 & 100 & 100 & 94 & 35 \\
\hline & & 3 & 100 & 94 & 80 & 58 & 100 & 100 & 56 & 0 \\
\hline
\end{tabular}

Table 6: Comparative studies on the basis of global performance indices for nonlinear RL circuit model

\begin{tabular}{|c|c|c|c|c|c|c|c|c|c|c|c|}
\hline \multirow{2}{*}{ Pr. } & \multirow{2}{*}{\multicolumn{2}{|c|}{ Operator }} & \multicolumn{3}{|c|}{ S:1 } & \multicolumn{3}{|c|}{ S:2 } & \multicolumn{3}{|c|}{ S:3 } \\
\hline & & & C:1 & $\mathrm{C}: 2$ & C:3 & C:1 & $C: 2$ & C:3 & C:1 & $C: 2$ & $C: 3$ \\
\hline \multirow{6}{*}{1} & \multirow{2}{*}{ GMAE } & $\begin{array}{l}\text { Values } \\
\end{array}$ & $4.75 \times 10^{-3}$ & $7.06 \times 10^{-3}$ & $9.76 \times 10^{-3}$ & $3.45 \times 10^{-3}$ & $2.59 \times 10^{-3}$ & $2.75 \times 10^{-3}$ & $5.68 \times 10^{-4}$ & $8.90 \times 10^{-4}$ & $1.23 \times 10^{-3}$ \\
\hline & & STD & $9.89 \times 10^{-4}$ & $4.22 \times 10^{-3}$ & $4.14 \times 10^{-3}$ & $9.75 \times 10^{-4}$ & $1.09 \times 10^{-3}$ & $9.79 \times 10^{-4}$ & $2.30 \times 10^{-4}$ & $4.23 \times 10^{-4}$ & $5.67 \times 10^{-4}$ \\
\hline & \multirow{2}{*}{$\mathbf{E G}_{\mathrm{NSE}}$} & Values & $2.66 \times 10^{-5}$ & $1.98 \times 10^{-4}$ & $1.99 \times 10^{-4}$ & $9.68 \times 10^{-6}$ & $6.44 \times 10^{-6}$ & $4.50 \times 10^{-6}$ & $2.30 \times 10^{-7}$ & $3.00 \times 10^{-6}$ & $1.15 \times 10^{-5}$ \\
\hline & & STD & $4.78 \times 10^{-4}$ & $1.64 \times 10^{-3}$ & $3.20 \times 10^{-3}$ & $1.57 \times 10^{-4}$ & $6.69 \times 10^{-5}$ & $5.40 \times 10^{-5}$ & $1.24 \times 10^{-6}$ & $1.33 \times 10^{-5}$ & $4.82 \times 10^{-5}$ \\
\hline & \multirow[b]{2}{*}{$\mathbf{E G}_{\mathrm{VAF}}$} & Values & $1.73 \times 10^{-4}$ & $1.73 \times 10^{-3}$ & $2.06 \times 10^{-3}$ & $6.25 \times 10^{-5}$ & $4.06 \times 10^{-5}$ & $2.75 \times 10^{-5}$ & $6.75 \times 10^{-7}$ & $9.40 \times 10^{-6}$ & $3.15 \times 10^{-5}$ \\
\hline & & STD & $7.35 \times 10^{-5}$ & $1.88 \times 10^{-4}$ & $3.67 \times 10^{-5}$ & $2.44 \times 10^{-5}$ & $1.06 \times 10^{-5}$ & $8.83 \times 10^{-6}$ & $4.23 \times 10^{-7}$ & $4.25 \times 10^{-6}$ & $1.76 \times 10^{-5}$ \\
\hline \multirow{6}{*}{2} & \multirow{2}{*}{ GMAE } & Values & $1.16 \times 10^{-2}$ & $1.16 \times 10^{-2}$ & $1.20 \times 10^{-2}$ & $5.10 \times 10^{-3}$ & $2.85 \times 10^{-3}$ & $2.98 \times 10^{-3}$ & $2.84 \times 10^{-3}$ & $3.79 \times 10^{-3}$ & $1.83 \times 10^{-3}$ \\
\hline & & STD & $1.25 \times 10^{-2}$ & $1.13 \times 10^{-2}$ & $1.10 \times 10^{-2}$ & $4.08 \times 10^{-3}$ & $2.40 \times 10^{-3}$ & $2.40 \times 10^{-3}$ & $2.33 \times 10^{-3}$ & $3.68 \times 10^{-3}$ & $1.62 \times 10^{-3}$ \\
\hline & \multirow{2}{*}{$\mathbf{E G}_{\text {NSE }}$} & Values & $1.16 \times 10^{-2}$ & $1.16 \times 10^{-2}$ & $1.20 \times 10^{-2}$ & $5.10 \times 10^{-3}$ & $2.85 \times 10^{-3}$ & $2.98 \times 10^{-3}$ & $2.84 \times 10^{-3}$ & $3.79 \times 10^{-3}$ & $1.83 \times 10^{-3}$ \\
\hline & & STD & $1.25 \times 10^{-2}$ & $1.13 \times 10^{-2}$ & $1.10 \times 10^{-2}$ & $4.08 \times 10^{-3}$ & $2.40 \times 10^{-3}$ & $2.40 \times 10^{-3}$ & $2.33 \times 10^{-3}$ & $3.68 \times 10^{-3}$ & $1.62 \times 10^{-3}$ \\
\hline & \multirow{2}{*}{ EGvaF } & Values & $3.42 \times 10^{-3}$ & $5.26 \times 10^{-3}$ & $1.42 \times 10^{-2}$ & $6.06 \times 10^{-4}$ & $1.75 \times 10^{-4}$ & $2.31 \times 10^{-4}$ & $2.91 \times 10^{-4}$ & $4.44 \times 10^{-4}$ & $8.02 \times 10^{-5}$ \\
\hline & & STD & $4.50 \times 10^{-3}$ & $6.05 \times 10^{-3}$ & $1.45 \times 10^{-2}$ & $5.05 \times 10^{-4}$ & $1.60 \times 10^{-4}$ & $1.96 \times 10^{-4}$ & $2.51 \times 10^{-4}$ & $4.57 \times 10^{-4}$ & $7.66 \times 10^{-5}$ \\
\hline
\end{tabular}

\section{Compliance with ethical standards}


- Conflict of interest: All the authors of the manuscript declared that there are no potential conflicts of interest.

- Human and animal rights statements: All the authors of the manuscript declared that there is no research involving human participants and/or animal.

- Informed consent: All the authors of the manuscript declared that there is no material that required informed consent.

\section{Appendix}

The numerical illustrations of results in case of $\mathrm{AC}$ excitation are given in Tables A1 and A2, while the graphics presented in Figs. A1 to A5. Appendix section is provided in Microsoft word document "Appendix.docx" which is submitted as a supplementary material.

\section{References}

[1] Raja, M.A.Z., Zameer, A., Kiani, A.K., Shehzad, A. and Khan, M.A.R., 2018. Nature-inspired computational intelligence integration with Nelder-Mead method to solve nonlinear benchmark models. Neural Computing and Applications, 29(4), pp.1169-1193.

[2] Fardad, K., Najafi, B., Ardabili, S.F., Mosavi, A., Shamshirband, S. and Rabczuk, T., 2018. Biodegradation of medicinal plants waste in an anaerobic digestion reactor for biogas production. Computers, Materials and Continua, 55(3), pp.318-392.

[3] Raja, M.A.Z., Mehmood, A., Niazi, S.A. and Shah, S.M., 2018. Computational intelligence methodology for the analysis of RC circuit modelled with nonlinear differential order system. Neural Computing and Applications, 30(6), pp.1905-1924.

[4] Ahmad, I., et al., 2018. Intelligent computing to solve fifth-order boundary value problem arising in induction motor models. Neural Computing and Applications, 29(7), pp.449-466.

[5] Liu, W., Chen, Z., Liu, J., Su, Z. and Chi, L., 2018. Full-Blind Delegating Private Quantum Computation. Computers, Materials and Continua, 56(2), pp.211-223.

[6] Soodi, H.A. and Vural, A.M., 2018. STATCOM Estimation Using Back-Propagation, PSO, Shuffled Frog Leap Algorithm, and Genetic Algorithm Based Neural Networks. Computational intelligence and neuroscience, 2018. https://doi.org/10.1155/2018/6381610.

[7] Raja, M.A.Z., Umar, M., Sabir, Z., Khan, J.A. and Baleanu, D., 2018. A new stochastic computing paradigm for the dynamics of nonlinear singular heat conduction model of the human head. The European Physical Journal Plus, 133(9), p.364.

[8] Mozaffari, A., Gorji-Bandpy, M., Samadian, P., Rastgar, R. and Kolaei, A.R., 2013. Comprehensive preference optimization of an irreversible thermal engine using Pareto based mutable smart bee algorithm and generalized regression neural network. Swarm and Evolutionary Computation, 9, pp.90-103.

[9] Yu, Z., Wang, Y., Zheng, B., Zheng, H., Wang, N. and Gu, Z., 2017. Underwater Inherent Optical Properties Estimation Using a Depth Aided Deep Neural Network. Computational intelligence and neuroscience, 2017. https://doi.org/10.1155/2017/8351232.

[10] Şahin, M. and Erol, R., 2018. Prediction of Attendance Demand in European Football Games: Comparison of ANFIS, Fuzzy Logic, and ANN. Computational intelligence and neuroscience, 2018. https://doi.org/10.1155/2018/5714872.

[11] Mall, S. and Chakraverty, S., 2017. Single layer Chebyshev neural network model for solving elliptic partial differential equations. Neural Processing Letters, 45(3), pp.825-840. 
[12] Tang, Y., Ji, J., Gao, S., Dai, H., Yu, Y. and Todo, Y., 2018. A pruning neural network model in credit classification analysis. Computational intelligence and neuroscience, 2018.. https://doi.org/10.1155/2018/9390410.

[13] Arqub, O.A., Mohammed, A.S., Momani, S. and Hayat, T., 2016. Numerical solutions of fuzzy differential equations using reproducing kernel Hilbert space method. Soft Computing, 20(8), pp.32833302.

[14] Yadav, N., McFall, K.S., Kumar, M. and Kim, J.H., 2018. A length factor artificial neural network method for the numerical solution of the advection dispersion equation characterizing the mass balance of fluid flow in a chemical reactor. Neural Computing and Applications, 30(3), pp.917-924.

[15] Arqub, O.A. and Abo-Hammour, Z., 2014. Numerical solution of systems of second-order boundary value problems using continuous genetic algorithm. Information sciences, 279, pp.396-415.

[16] Munir, A., et al., 2019. Intelligent computing approach to analyze the dynamics of wire coating with Oldroyd 8-constant fluid. Neural Computing and Applications, 31(3), pp.751-775.

[17] Ahmad, I., et al., 2018. Neuro-evolutionary computing paradigm for Painlevé equation-II in nonlinear optics. The European Physical Journal Plus, 133(5), p.184.

[18] Raja, M.A.Z., Shah, Z., Manzar, M.A., Ahmad, I., Awais, M. and Baleanu, D., 2018. A new stochastic computing paradigm for nonlinear Painlevé II systems in applications of random matrix theory. The European Physical Journal Plus, 133(7), p.254.

[19] Khan, J.A., et al., 2015. Design and application of nature inspired computing approach for nonlinear stiff oscillatory problems. Neural Computing and Applications, 26(7), pp.1763-1780.

[20] Arqub, O.A., Al-Smadi, M., Momani, S. and Hayat, T., 2017. Application of reproducing kernel algorithm for solving second-order, two-point fuzzy boundary value problems. Soft Computing, 21(23), pp.7191-7206.

[21] Mehmood, A., et al., 2018. Intelligent computing to analyze the dynamics of Magnetohydrodynamic flow over stretchable rotating disk model. Applied Soft Computing, 67, pp.8-28.

[22] Momani, S., Abo-Hammour, Z.S. and Alsmadi, O.M., 2016. Solution of inverse kinematics problem using genetic algorithms. Applied Mathematics \& Information Sciences, 10(1), p.225.

[23] Mehmood, A., et al., 2018. Design of neuro-computing paradigms for nonlinear nanofluidic systems of MHD Jeffery-Hamel flow. Journal of the Taiwan Institute of Chemical Engineers, 91, pp.57-85.

[24] Raja, M.A.Z., Shah, A.A., Mehmood, A., Chaudhary, N.I. and Aslam, M.S., 2018. Bio-inspired computational heuristics for parameter estimation of nonlinear Hammerstein controlled autoregressive system. Neural Computing and Applications, 29(12), pp.1455-1474.

[25] Raja, M.A.Z., 2014. Solution of the one-dimensional Bratu equation arising in the fuel ignition model using ANN optimised with PSO and SQP. Connection Science, 26(3), pp.195-214.

[26] Arqub, O.A., 2017. Adaptation of reproducing kernel algorithm for solving fuzzy Fredholm-Volterra integrodifferential equations. Neural Computing and Applications, 28(7), pp.1591-1610.

[27] Raja, M.A.Z., Shah, F.H., Khan, A.A. and Khan, N.A., 2016. Design of bio-inspired computational intelligence technique for solving steady thin film flow of Johnson-Segalman fluid on vertical cylinder for drainage problems. Journal of the Taiwan Institute of Chemical Engineers, 60, pp.59-75.

[28]. Raja, M.A.Z., Samar, R., Alaidarous, E.S. and Shivanian, E., 2016. Bio-inspired computing platform for reliable solution of Bratu-type equations arising in the modeling of electrically conducting solids. Applied Mathematical Modelling, 40(11-12), pp.5964-5977. 
[29] Raja, M.A.Z., Ahmed, T. and Shah, S.M., 2017. Intelligent computing strategy to analyze the dynamics of convective heat transfer in MHD slip flow over stretching surface involving carbon nanotubes. Journal of the Taiwan Institute of Chemical Engineers, 80, pp.935-953.

[30] Ahmad, I., et al., 2016. Bio-inspired computational heuristics to study Lane-Emden systems arising in astrophysics model. SpringerPlus, 5(1), p.1866.

[31] Raja, M.A.Z., Shah, F.H., Tariq, M. and Ahmad, I., 2018. Design of artificial neural network models optimized with sequential quadratic programming to study the dynamics of nonlinear Troesch's problem arising in plasma physics. Neural Computing and Applications, 29(6), pp.83-109.

[32] Sabir, Z., et al., 2018. Neuro-heuristics for nonlinear singular Thomas-Fermi systems. Applied Soft Computing, 65, pp.152-169.

[33] Raja, M.A.Z., Shah, F.H., Alaidarous, E.S. and Syam, M.I., 2017. Design of bio-inspired heuristic technique integrated with interior-point algorithm to analyze the dynamics of heartbeat model. Applied Soft Computing, 52, pp.605-629.

[34] Raja, M.A.Z., Asma, K. and Aslam, M.S., 2018. Bio-inspired computational heuristics to study models of HIV infection of CD4+ T-cell. International Journal of Biomathematics, 11(02), p.1850019.

[35] Jafarian, A., Mokhtarpour, M. and Baleanu, D., 2017. Artificial neural network approach for a class of fractional ordinary differential equation. Neural Computing and Applications, 28(4), pp.765-773.

[36] Zafer ur Rehman, et al., 2018. Design of reduced search space strategy based on integration of Nelder-Mead method and pattern search algorithm with application to economic load dispatch problem. Neural Computing and Applications, 30(12), pp.3693-3705.

[37] Ara, A., et al., 2018. Wavelets optimization method for evaluation of fractional partial differential equations: an application to financial modelling. Advances in Difference Equations, 2018(1), p.8.

[38] Raja, M.A.Z., Zameer, A., Khan, A.U. and Wazwaz, A.M., 2016. A new numerical approach to solve Thomas-Fermi model of an atom using bio-inspired heuristics integrated with sequential quadratic programming. SpringerPlus, 5(1), p.1400.

[39] Ahmad, I., et al., 2017. Neural network methods to solve the Lane-Emden type equations arising in thermodynamic studies of the spherical gas cloud model. Neural Computing and Applications, 28(1), pp.929-944.

[40] Mall, S. and Chakraverty, S., 2015. Numerical solution of nonlinear singular initial value problems of Emden-Fowler type using Chebyshev Neural Network method. Neurocomputing, 149, pp.975-982.

[41] Masood, Z., et al., 2017. Design of Mexican Hat Wavelet neural networks for solving Bratu type nonlinear systems. Neurocomputing, 221, pp.1-14.

[42] Majeed, K., et al., 2017. A genetic algorithm optimized Morlet wavelet artificial neural network to study the dynamics of nonlinear Troesch's system. Applied Soft Computing, 56, pp.420-435.

[43] Lodhi, S., et al., 2019. Fractional neural network models for nonlinear Riccati systems. Neural Computing and Applications, 31(1), pp.359-378.

[44] Raja, M.A.Z., Khan, J.A., Zameer, A., Khan, N.A. and Manzar, M.A., 2019. Numerical treatment of nonlinear singular Flierl-Petviashivili systems using neural networks models. Neural Computing and Applications, 31(7), pp 2371-2394.

[45] Raja, M.A.Z., Samar, R., Manzar, M.A. and Shah, S.M., 2017. Design of unsupervised fractional neural network model optimized with interior point algorithm for solving Bagley-Torvik equation. Mathematics and Computers in Simulation, 132, pp.139-158. 
[46] Raja, M.A.Z., Ahmad, I., Khan, I., Syam, M.I. and Wazwaz, A.M., 2017. Neuro-heuristic computational intelligence for solving nonlinear pantograph systems. Frontiers of Information Technology \& Electronic Engineering, 18(4), pp.464-484.

[47] Khan, J.A., et al., 2011. Novel approach for a van der pol oscillator in the continuous time domain. Chinese Physics Letters, 28(11), p.110205.

[48] Ahmad, I., et al., 2019. Heuristic computational intelligence approach to solve nonlinear multiple singularity problem of sixth Painlevé equation. Neural Computing and Applications, 31(1), pp.101-115.

[49] Köksal, M. and Herdem, S., 2002. Analysis of nonlinear circuits by using differential Taylor transform. Computers \& Electrical Engineering, 28(6), pp.513-525.

[50] J. H. Holland, "Adaptation in natural and artificial systems," Ann arbor, MI, University of Michigan press, 1975.

[51] Shi, L., Wan, Y., Gao, X. and Wang, M., 2018. Feature Selection for Object-Based Classification of High-Resolution Remote Sensing Images Based on the Combination of a Genetic Algorithm and Tabu Search. Computational intelligence and neuroscience, 2018. https://doi.org/10.1155/2018/6595792.

[52] Khan, W.U., et al., 2018. Backtracking search integrated with sequential quadratic programming for nonlinear active noise control systems. Applied Soft Computing, 73, pp.666-683.

[53] Raja, M.A.Z., Mehmood, J., Sabir, Z., Nasab, A.K. and Manzar, M.A., 2019. Numerical solution of doubly singular nonlinear systems using neural networks-based integrated intelligent computing. Neural Computing and Applications, 31(3), pp.793-812.

[54] Zaman, F., Qureshi, I.M., Rehman, A.U. and Tanoli, S.A.K., 2015. Multiple target localization with bistatic radar using heuristic computational intelligence techniques. International Journal of Antennas and Propagation, 2015. https://doi.org/10.1155/2015/982967.

[55] Zaman, F., 2017. Joint angle-amplitude estimation for multiple signals with L-structured arrays using bioinspired computing. Wireless Communications and Mobile Computing, 2017. https://doi.org/10.1155/2017/9428196.

[56] Hussain, A., Muhammad, Y.S., Nauman Sajid, M., Hussain, I., Mohamd Shoukry, A. and Gani, S., 2017. Genetic algorithm for traveling salesman problem with modified cycle crossover operator. Computational intelligence and neuroscience, 2017.

https://doi.org/10.1155/2017/7430125.

[57] Zameer, A., et al., 2019. Bio-inspired heuristics for layer thickness optimization in multilayer piezoelectric transducer for broadband structures. Soft Computing, 23(10), pp.3449-3463.

[58] Inthachot, M., Boonjing, V. and Intakosum, S., 2016. Artificial neural network and genetic algorithm hybrid intelligence for predicting thai stock price index trend. Computational intelligence and neuroscience, 2016. https://doi.org/10.1155/2016/3045254.

[59] Bandyopadhyay, A. and Kar, S., 2018. System of type-2 fuzzy differential equations and its applications. Neural Computing and Applications, in press

https://doi.org/10.1007/s00521-018-3380-x

[60] Boggs, Paul T., and Jon W. Tolle. "Sequential quadratic programming." Acta numerica 4 (1995): 151.

[61] Nocedal, J. and Wright, S.J., 2006. Sequential quadratic programming. Numerical optimization, pp.529-562. 
[62] Singh, G., Rattan, M., Gill, S.S. and Mittal, N., 2019. Hybridization of water wave optimization and sequential quadratic programming for cognitive radio system. Soft Computing, 23(17), pp.7991-8011.

[63] Liao, H., Wu, W. and Fang, D., 2018. The reduced space Sequential Quadratic Programming (SQP) method for calculating the worst resonance response of nonlinear systems. Journal of Sound and Vibration, 425, pp.301-323.

[64] Nash, J.E., ad Sutcliffe, JV 1970. River flow forecasting through conceptual models part I-A discussion of principles. Journal of Hydrology, 10(3), pp.282-290.

[65] Ghasemi, H., Park, H.S. and Rabczuk, T., 2018. A multi-material level set-based topology optimization of flexoelectric composites. Computer Methods in Applied Mechanics and Engineering, 332, pp.47-62.

[66] Ghasemi, H., Park, H.S. and Rabczuk, T., 2017. A level-set based IGA formulation for topology optimization of flexoelectric materials. Computer Methods in Applied Mechanics and Engineering, 313, pp.239-258. 